\title{
RECENT ADVANCES IN COMPUTATIONAL CONFORMAL GEOMETRY
}

\author{
XIANFENG DAVID GU*, FENG LUO ${ }^{\dagger}$, AND SHING-TUNG YAU Y $^{\ddagger}$
}

\begin{abstract}
Computational conformal geometry focuses on developing the computational methodologies on discrete surfaces to discover conformal geometric invariants. In this work, we briefly summarize the recent developments for methods and related applications in computational conformal geometry. There are two major approaches, holomorphic differentials and curvature flow. Holomorphic differential method is a linear method, which is more efficient and robust to triangulations with lower quality. Curvature flow method is nonlinear and requires higher quality triangulations, but it is more flexible. The conformal geometric methods have been broadly applied in many engineering fields, such as computer graphics, vision, geometric modeling and medical imaging. The algorithms are robust for surfaces scanned from real life, general for surfaces with different topologies. The efficiency and efficacy of the algorithms are demonstrated by the experimental results.
\end{abstract}

Keywords: Computational Conformal Geometry, Holomorphic Differential, Curvature Flow

1. Introduction. Computational conformal geometry focuses on developing the computational methodologies on discrete surfaces to discover conformal geometric invariants. Computational conformal geometry is an emerging field, which combines differential geometry, algebraic topology, complex analysis, Riemann surface theory, algebraic geometry with computer science. It has broad applications in many fields in both pure theoretic research, such as mathematics, theoretic physics, and engineering applications, such as mechanics, computer graphics, computer vision, geometric modeling, network and medical imaging.

Classical computational complex analysis focuses on the mappings among domains on the complex plane $\mathbb{C}$. Classical methods for constructing conformal mappings include Schwarz-Christoffel maps, osculation method, polynomial expansion method, circle packing method and many other methods. For details, we refer readers to [1] and [2] for a more thorough discussion.

With the development of 3D data acquisition technologies, huge amount of surface and volumetric data have been accumulated. For example, the state of art of 3D scanner based on phase shifting technology can capture dynamic surfaces with a quarter of million samples per frame, at the frame rate as high as 180 per second. Figure 1 illustrates one example of the acquired human face surface. The geometric surfaces are stored and represented in computers as polyhedral surfaces, most commonly simplicial complexes with a piecewise linear embedding in the Euclidean space,

\footnotetext{
* Computer Science Department, State University of New York at Stony Brook, Stony Brook, NY USA.

${ }^{\dagger}$ Mathematics Department, Rutgers University, Piscataway, NJ USA.

${ }^{\ddagger}$ Mathematics Department, Harvard University, Cambridge, MA USA.
} 


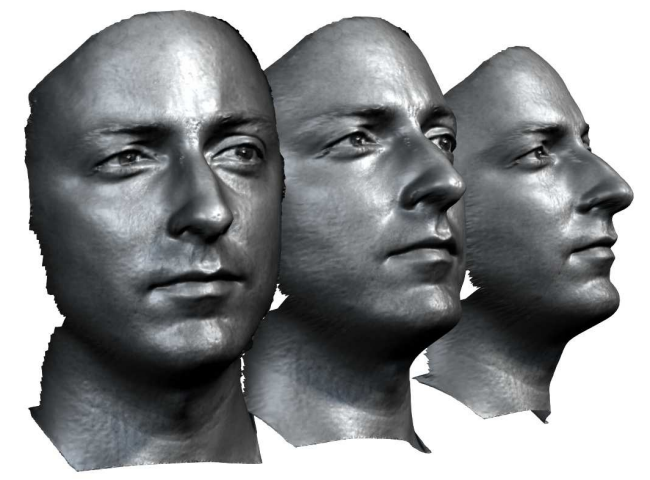

FIG. 1. Geometric surfaces captured by 3D scanner based on phase shifting technology.

or triangular meshes in engineering field as shown in Figure 2.

The demands for process discrete surfaces accurately and efficiently are pressing. Rigorous algorithms for surface matching, registration, classification have fundamental importance in almost every engineering field. It also becomes great challenges for mathematician to generalize the geometric theories from smooth manifolds to discrete settings.

With the great efforts of both mathematicians and computer scientists, major breakthroughs have been made in the last several years. Several theoretic frameworks have been systematically developed. The discrete counter parts of many fundamental theorems in classical conformal geometry have been discovered. Many computational algorithms have been invented and applied for engineering and medicine fields. There are still many profound facts in conformal geometry, the discretization method and the computational strategy are still widely open. Furthermore, the urge of practical applications have advanced the computational algorithms of this field, but the theoretic foundations need to be rigorously laid down in near future.

Generally speaking, there are two major approaches for computing conformal structures, one is along the harmonic analysis approach to construct various holomorphic or meromorphic differentials on the surface, then build conformal mappings, quasi-conformal mappings based on the differential forms; the other is along the surface Ricci curvature flow approach to design conformal Riemannian metrics which satisfy the prescribed curvatures. These two approaches both have advantages and disadvantages, and they are closely related. This work focuses on the recent developments of these approaches and their applications in practice. 

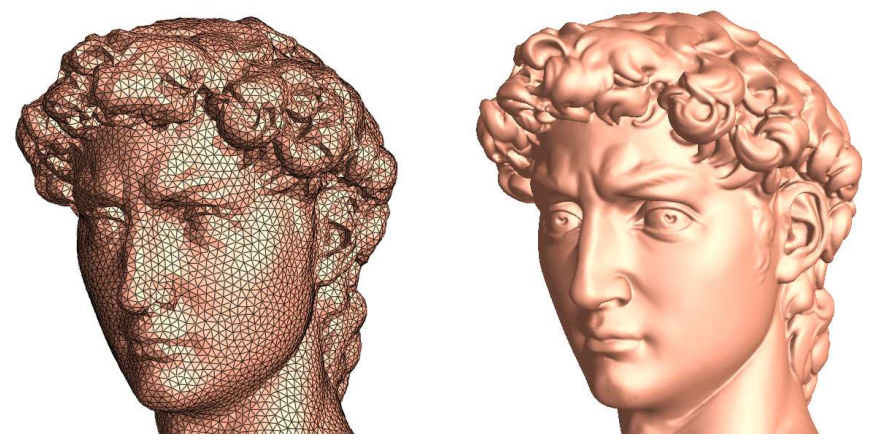

FIG. 2. Surfaces are represented as triangular meshes on computers.

2. Previous Work. Conventional computational complex analysis methods focus on conformal mappings on planar domains. A thorough introduction to the conventional methods can be found in the books [1] and [2].

Recently, with the development of digital scanning technology, computing conformal mappings between surfaces becomes more and more important. In computer graphics and discrete mathematics, much sound research has focused on discrete conformal mappings.

2.1. Holomorphic Differentials. The computational method of current work is mainly based on harmonic maps and holomorphic differential forms. Here, we briefly overview most related work, and refer readers to [3] and [4] for thorough surveys.

Discrete harmonic maps were constructed in [5], where the cotan formula was introduced. First order finite element approximations of the Cauchy-Riemann equations were introduced by Levy et al. [6]. Discrete intrinsic parameterization by minimizing Dirichlet energy was introduced by [7]. Mean value coordinates were introduced in [8] to compute generalized harmonic maps; Discrete spherical conformal mappings are used in [9] and [10].

Discrete holomorphic forms are introduced by Gu and Yau [11] to compute global conformal surface parameterizations for high genus surfaces. Another approach of discrete holomorphy was introduced in [12] using discrete exterior calculus [13]. The problem of computing optimal holomorphic 1-forms to reduce area distortion was considered in [14]. Gortler et al. [15] generalized 1-forms to the discrete case, using them to parameterize genus one meshes. Tong et al. [16] generalized the 1-form method to incorporate cone singularities. Discrete one-forms have been applied for meshing point clouds in [17], surface tiling [18], surface quadrangulation [19]. Holomorphic 1-form method has been applied for virtual colonoscopy [20]. The colon surface is reconstructed from MRI images, and conformally mapped to the planar rectangle. This improves the efficiency and accuracy for detecting polyps. Conformal mapping is used for brain cortex surface morphology study in [10]. By mapping brain sur- 
faces to spheres, cortex surface registration and comparison become straightforward. Holomorphic 1-form method has also been applied in computer vision [21, 22] for 3D shape matching, recognition and stitching. In geometric modeling field, constructing splines on general surfaces is one of the most fundamental problems. It is proven in [23] that if the surface has an affine structure, then spline can be generalized to it directly. Holomorphic 1-forms can be applied for computing the affine structures of general surfaces.

\subsection{Curvature Flow.}

2.2.1. The Ricci Flow on Surfaces. The Ricci flow was introduced by R. Hamilton in a seminal paper [24] for Riemannian manifolds of any dimension. The Ricci flow has revolutionized the study of geometry of surfaces and 3-manifolds and has inspired huge research activities in geometry. In particular, it leads to a proof of the 3-dimensional Poincaré conjecture. In the paper [25], Hamilton used the 2dimensional Ricci flow to give a proof of the uniformization theorem for surfaces of positive genus. This leads a way for potential applications to computer graphics.

There are many ways to discretize smooth surfaces. The one which is particularly related to a discretization of conformality is the circle packing metric introduced by Thurston [26]. The notion of circle packing has appeared in the work of Koebe [27]. Thurston conjectured in [28] that for a discretization of the Jordan domain in the plane, the sequence of circle packings converge to the Riemann mapping. This was proved by Rodin and Sullivan [29].

Colin de Verdiere [30] established the first variational principle for circle packing and proved Thurston's existence of circle packing metrics. This paved a way for a fast algorithmic implementation of finding the circle packing metrics, such as the one by Collins and Stephenson [31]. In [32], Chow and Luo generalized Colin de Verdiere's work and introduced the discrete Ricci flow and discrete Ricci energy on surfaces. They proved a general existence and convergence theorem for the discrete Ricci flow and proved that the Ricci energy is convex. The algorithmic implementation of the discrete Ricci flow was carried out by Jin et al. [33].

Another related discretization method is called circle pattern; it considers both the combinatorics and the geometry of the original mesh, and can be looked as a variant to circle packings. Circle pattern was proposed by Bowers and Hurdal [34], and has been proven to be a minimizer of a convex energy by Bobenko and Springborn [35]. An efficient circle pattern algorithm was developed by Kharevych et al. [36].

2.2.2. The Yamabe Flow on Surfaces. The Yamabe problem aims at finding a conformal metric with constant scalar curvature for compact Riemannian manifolds. The first proof (with flaws) was given by Yamabe [37], which was corrected and extended to a complete proof by several researchers including Trudinger [38], Aubin [39] and Schoen [40]. A comprehensive survey on this topic was given by Lee and 
Parker in [41].

In [42] Luo studied the discrete Yamabe flow on surfaces. He introduced a notion of discrete conformal change of polyhedral metric, which plays a key role in developing the discrete Yamabe flow and the associated variational principle in the field. Based on the discrete conformal class and geometric consideration, Luo gave the discrete Yamabe energy as an integration of a differential 1-form and proved that this energy is a locally convex function. He also deduced from it that the curvature evolution of the Yamabe flow is a heat equation.

In a very nice recent work of Springborn et al. [43] they were able to identify the Yamabe energy introduced by Luo with the Milnor-Lobachevsky function and the heat equation for the curvature evolution with the cotangent Laplace equation. They constructed an algorithm based on their explicit formula. Another recent work by $\mathrm{Gu}$ et al. [44], which used the original discrete Yamabe energy from [42], has produced an equally efficient algorithm in finding the discrete conformal metrics. In addition, discrete hyperbolic Yamabe Flow was presented in [45] for computing hyperbolic structure and the canonical homotopy class representative.

3. Theoretic Background. This section review the preliminary theoretic background for conformal geometry.

3.1. Harmonic Maps. Suppose $f: S \rightarrow \mathbb{R}$ is a function defined on a surface $S$, the harmonic energy of $f$ is defined as

$$
E(f)=\int_{S}|\nabla f|^{2} d s
$$

where $\nabla f$ is the gradient of $f$. A harmonic function is a critical point of the harmonic energy. Harmonic function satisfies the following Laplace equation

$$
\Delta f=0,
$$

where $\Delta$ is the Laplace-Beltrami operator. If $S$ is a domain on the Euclidean plane, then the Laplace-Beltrami operator has the form

$$
\Delta=\frac{\partial^{2}}{\partial x^{2}}+\frac{\partial^{2}}{\partial y^{2}}
$$

A harmonic map between two surfaces can be defined similarly, which is a critical point of the harmonic energy. Suppose $\phi: S_{1} \rightarrow S_{2}$ is a harmonic map, $S_{2}$ is a convex planar domain, and the restriction of $\phi$ on the boundaries $\phi_{\partial S_{1}}: \partial S_{1} \rightarrow \partial S_{2}$ is a homeomorphism, then $\phi$ is a diffeomorphism in the interior. Figure 3 shows one such kind of map.

3.2. Conformal Mappings. Let $S_{1}$ and $S_{2}$ be two surfaces with Riemannian metrics $\mathbf{g}_{1}$ and $\mathbf{g}_{2}, \phi:\left(S_{1}, \mathbf{g}_{1}\right) \rightarrow\left(S_{2}, \mathbf{g}_{2}\right)$ be a homeomorphism between them. We say $\phi$ is conformal, if it preserves angles. In details, as shown in Fig. 4, let 

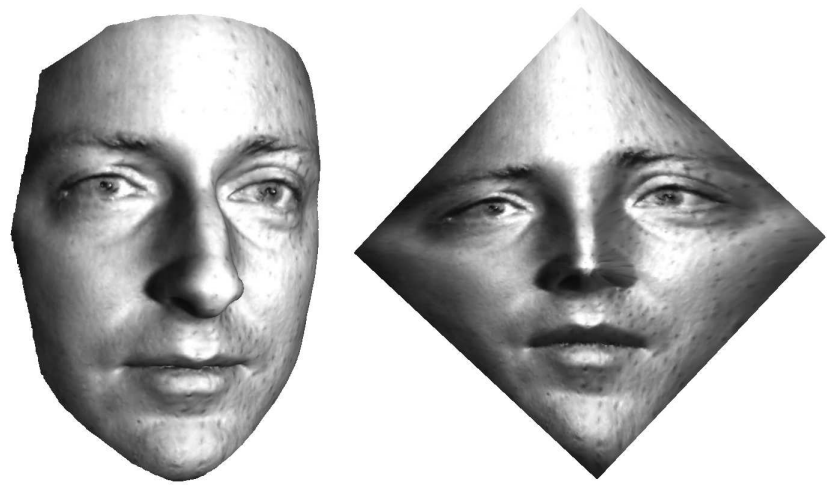

FIG. 3. A harmonic map from a face surface to a planar convex domain.

$\gamma_{1}, \gamma_{2}:[0,1] \rightarrow S_{1}$ be two arbitrary curves on $S_{1}$, intersecting at the point $p$, the angle between the two tangent vectors $\frac{d \gamma_{1}}{d t}(p)$ and $\frac{d \gamma_{2}}{d t}(p)$ equal to $\theta$. Therefore $\phi \circ \gamma_{1}(t)$ and $\phi \circ \gamma_{2}(t)$ are two curves on $S_{2}$, intersecting at $\phi(p)$. Then their intersection angle also equals to $\theta$.
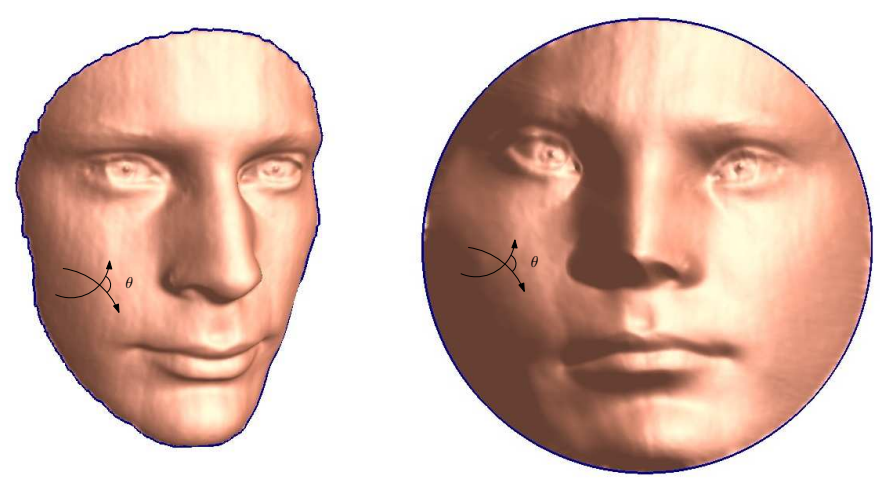

FIG. 4. Conformal mappings preserve angles.

Locally, conformal mapping is a scaling transformation, it preserves local shapes. For example, it maps infinitesimal circles to infinitesimal circles. As shown in Fig. 5 frame (a), the bunny surface is mapped to the plane via a conformal mapping. A circle packing is defined on the plane, and pulled back onto the bunny surface, and all small circles are preserved. If we put a checkerboard on the plane, then on the bunny surface, all the right angles of the checkers are well preserved, as illustrated in the same figure frame (a).

Mathematically, the pull back metric induced by $\phi$ differs from the original metric by a scaling function, which is called the conformal factor and measures the area distortion.

$$
\phi_{*}\left(\mathbf{g}_{2}\right)=e^{2 u} \mathbf{g}_{1} .
$$




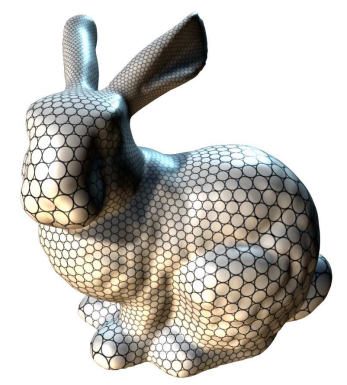

(a)Circle packing

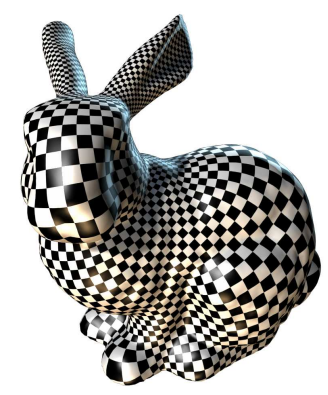

(b) Checker board

Fig. 5. Conformal texture mapping.

Conformal mappings have deep relation with complex analysis. Conformal mappings between two planar domains can be represented as holomorphic functions. Let $\phi: \mathbb{C} \rightarrow \mathbb{C}$ be a complex function, $\phi: z \rightarrow w$, where $z=x+i y, w=u+i v$, then $\phi$ is holomorphic, if it satisfies the following Riemann-Cauchy equation

$$
\frac{\partial u}{\partial x}=\frac{\partial v}{\partial y}, \frac{\partial u}{\partial y}=-\frac{\partial v}{\partial x}
$$

If $\phi$ is invertible, the inverse is also holomorphic, then $\phi$ is bi-holomorphic.

3.3. Conformal Structure. Let $S$ be a topological surface, $U_{\alpha}$ be an open set on $S, \phi_{\alpha}: U_{\alpha} \rightarrow \mathbb{C}$ be a homeomorphism, then $\left(U_{\alpha}, \phi_{\alpha}\right)$ is a local chart of $S$. Suppose $\left(U_{\beta}, \phi_{\beta}\right)$ is another local chart overlapping with $\left(U_{\alpha}, \phi_{\alpha}\right)$, the transition function $\phi_{\alpha \beta}: \phi_{\alpha}\left(U_{\alpha} \cap U_{\beta}\right) \rightarrow \phi_{\beta}\left(U_{\alpha} \cap U_{\beta}\right)$ is given by $\phi_{\beta} \circ \phi_{\alpha}^{-1}$. An atlas is a collection of local charts $\left\{\left(U_{\alpha}, \phi_{\alpha}\right)\right\}$, such that the union of the charts cover the surface, as shown in Fig. 6.

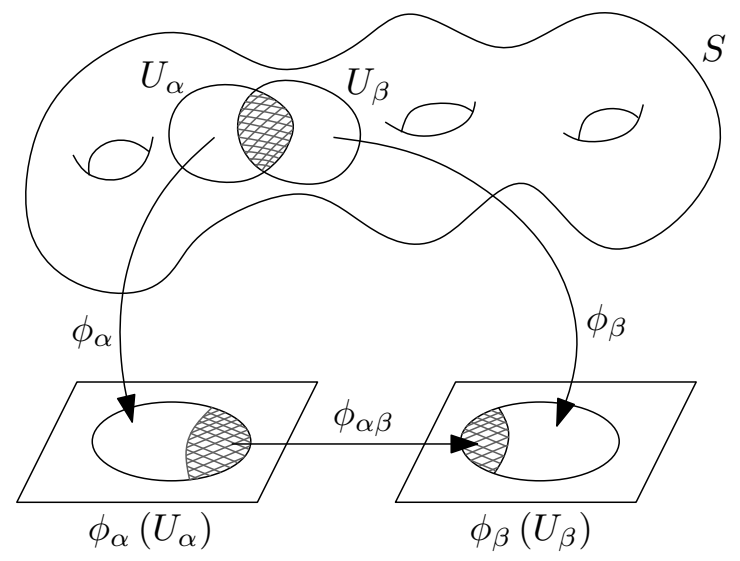

FIG. 6. Conformal structure.

An atlas is a conformal atlas if all its transition functions are biholomorphic. 
Two conformal atlas are equivalent if their union is still a conformal atlas. Each equivalence class of conformal atlases is called a conformal structure of the surface. A surface with a conformal structure is called a Riemann surface.

If a Riemann surface $S$ has a Riemannian metric $\mathbf{g}$, then we say its conformal structure is compatible with the metric if local representation of the metric on a chart $\left(U_{\alpha}, \phi_{\alpha}\right)$

$$
\mathbf{g}=e^{2 u} d z_{\alpha} d \bar{z}_{\alpha}
$$

where $z_{\alpha}$ is the local complex parameter. We also call such local complex parameter as isothermal coordinates. The Laplace-Beltrami operator $\Delta$ on an isothermal coordinates has a simple representation

$$
\Delta=\frac{1}{e^{2 u\left(x_{\alpha}, y_{\alpha}\right)}}\left(\frac{\partial^{2}}{\partial x_{\alpha}^{2}}+\frac{\partial^{2}}{\partial y_{\alpha}^{2}}\right) .
$$

All oriented metric surfaces are Riemann surfaces and with isothermal coordinates. Therefore conformal geometric concepts and methods are general to all surfaces in real life. Figure 7 shows the isothermal coordinates on surfaces from real life.
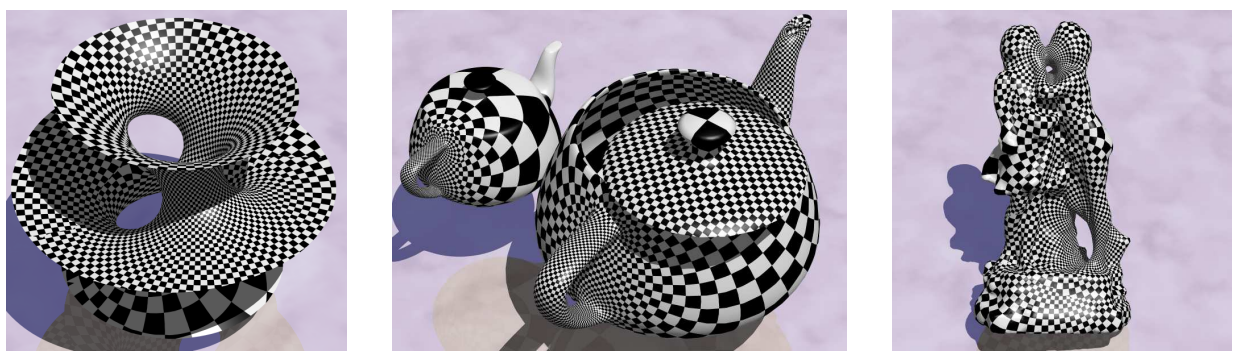

FIG. 7. Conformal structures (isothermal coordinates) for surfaces in real life.

Suppose $\left(S_{1}, \mathcal{A}_{1}\right)$ and $\left(S_{2}, \mathcal{A}_{2}\right)$ are two Riemann surfaces, $\mathcal{A}_{i}$ 's are their conformal structures, $\left(U_{\alpha}, \phi_{\alpha}\right)$ is a local chart of $\mathcal{A}_{1}$ and $\left(V_{\beta}, \psi_{\beta}\right)$ is a local chart of $\mathcal{A}_{2}$, then $\phi: S_{1} \rightarrow S_{2}$ is a conformal map if and only if

$$
\psi_{\beta} \circ \phi \circ \phi_{\alpha}^{-1}: \phi_{\alpha}\left(U_{\alpha}\right) \rightarrow \psi_{\beta}\left(V_{\beta}\right)
$$

is biholomorphic. A conformal map preserves angles.

3.4. Holomorphic Differentials. Differential forms play important role in conformal geometry. A function $f: S \rightarrow \mathbb{R}$ is called a 0 -form. The exterior differential operator $d$ is the generalization of conventional grad, curl and divergence operators. The exterior differentiation of a 0 -form is its gradient. Let $\left(U_{\alpha}, \phi_{\alpha}\right)$ be a local chart with local parameters $\left(x_{\alpha}, y_{\alpha}\right)$, then

$$
d_{0} f=\frac{\partial f}{\partial x_{\alpha}} d x_{\alpha}+\frac{\partial f}{\partial y_{\alpha}} d y_{\alpha} .
$$


A differential 1-form has local representation

$$
\omega=f_{\alpha} d x_{\alpha}+g_{\alpha} d y_{\alpha}
$$

and also has local representation $f_{\beta} d x_{\beta}+g_{\beta} d y_{\beta}$, such that

$$
\left(\begin{array}{ll}
f_{\alpha} & g_{\alpha}
\end{array}\right)\left(\begin{array}{ll}
\frac{\partial x_{\alpha}}{\partial x_{\beta}} & \frac{\partial x_{\alpha}}{\partial y_{\beta}} \\
\frac{\partial y_{\alpha}}{\partial x_{\beta}} & \frac{\partial y_{\alpha}}{\partial y_{\beta}}
\end{array}\right)=\left(\begin{array}{ll}
f_{\beta} & g_{\beta}
\end{array}\right) .
$$

The exterior differentiation of a 1-form is its circulation

$$
d_{1} \omega=\left(\frac{\partial g}{\partial x_{\alpha}}-\frac{\partial f}{\partial y_{\alpha}}\right) d x_{\alpha} \wedge d y_{\alpha} .
$$

A differential 2-form has local representation

$$
\Omega=f_{\alpha} d x_{\alpha} \wedge d y_{\alpha}
$$

On another chart $\left(x_{\beta}, y_{\beta}\right)$ and its associated representation $\Omega=f_{\beta} d x_{\beta} \wedge d y_{\beta}$, the two $f_{\alpha}$ and $f_{\beta}$ satisfy

$$
f_{\alpha}\left|\begin{array}{ll}
\frac{\partial x_{\alpha}}{\partial x_{\beta}} & \frac{\partial x_{\alpha}}{\partial y_{\beta}} \\
\frac{\partial y_{\alpha}}{\partial x_{\beta}} & \frac{\partial y_{\alpha}}{\partial y_{\beta}}
\end{array}\right|=f_{\beta} .
$$

The exterior differentiation of a 2 -form is zero, $d_{2} \Omega=0$.

Let $\omega$ be a $k$-form, if $d_{k} \omega=0, \omega$ is called a closed form. If there exists a $(k-1)$ form $\tau$, such that $\omega=d_{k-1} \tau$, then $\omega$ is called an exact form. A fundamental fact is that

$$
d_{k} \circ d_{k-1} \equiv 0, k=1,2
$$

therefore all exact forms are closed. The difference between exact forms and closed forms conveys the topological information of the surface. The de Rham cohomology group is defined as

$$
H^{k}(S, \mathbb{R})=\frac{\operatorname{Ker} d_{k}}{\operatorname{Img} d_{k-1}} .
$$

If two closed $k$-forms, $\omega_{1}, \omega_{2}$, are cohomologous, then they differ by an exact form $d \tau=\omega_{1}-\omega_{2}$, for some $(k-1)$-form $\tau$.

Let $\Omega_{0}$ be the area element on $S$ with local representation $d x_{\alpha} \wedge d y_{\alpha}, \omega$ be a $k$-form. The Hodge star operator converts $\omega$ to a $(n-k)$-form, ${ }^{*} \omega$, such that

$$
\omega \wedge^{*} \omega=\Omega_{0}
$$

We say ${ }^{*} \omega$ is conjugate to $\omega$.

The co-differentiation operator $\delta$ is defined as

$$
\delta=* d * .
$$


A harmonic form $\omega$ satisfies the following condition

$$
d \omega=0, \delta \omega=0
$$

A holomorphic 1-form is a pair of harmonic 1-forms, the imaginary part is conjugate to the real part:

$$
\tau=\omega+i^{*} \omega
$$

On the conformal atlas, $\tau$ has local representation on $\left(U_{\alpha}, \phi_{\alpha}\right)$ with local parameter $z_{\alpha}$

$$
\tau=f_{\alpha}\left(z_{\alpha}\right) d z_{\alpha}
$$

where $f_{\alpha}$ is a holomorphic function. On another chart $\left(U_{\beta}, \phi_{\beta}\right)$ with local parameter $z_{\beta}, \tau=f_{\beta} d z_{\beta}$, such that

$$
f_{\alpha} \frac{d z_{\alpha}}{d z_{\beta}}=f_{\beta}
$$

Hodge theorem claims that each cohomologous class has a unique harmonic 1form. Therefore the group of all harmonic 1-forms is isomorphic to the first cohomology group $H^{1}(S, \mathbb{R})$. Also, the group of all holomorphic 1-forms is isomorphic to $H^{1}(S, \mathbb{R})$.

3.5. Surface Ricci Flow. Let $S$ be a topological surface. Consider all Riemannian metrics on $S$. Two metrics $\mathbf{g}_{1}, \mathbf{g}_{2}$ are conformal equivalent, if there is a function $u: S \rightarrow \mathbb{R}$ defined on the surface, such that $\mathbf{g}_{2}=e^{2 u} \mathbf{g}_{1}$. Each conformal equivalence class of Riemannian metrics is a conformal structure of $S$.

The Gaussian curvatures determined by $\mathbf{g}_{1}$ and $\mathbf{g}_{2}$ are denoted as $K_{1}$ and $K_{2}$, they are related by the following Yamabe equation

$$
K_{2}=\frac{1}{e^{2 u}}\left(-\Delta u+K_{1}\right)
$$

where $\Delta$ is the Laplace-Beltrami operator determined by the metric $\mathbf{g}_{1}$.

Hamilton introduced surface Ricci flow

$$
\frac{d g_{i j}}{d t}=-K g_{i j}
$$

During the flow, if the total area is preserved, then the Gaussian curvature will evolve according to a heat diffusion process. Hamilton and Chow together proved that surface Ricci flow converges to a special metric, whose Gaussian curvature is constant everywhere. In fact, their proofs give another approach for the Poincaré uniformization theorem, which states that for any metric surface $(S, \mathbf{g})$, there exists a metric conformal to the original metric, that induces constant Gaussian curvature. As shown in Fig. 8, all closed surfaces can be conformally deform to three canonical shapes, the unit sphere $\mathbb{S}^{2}$, the plane $\mathbb{E}^{2}$ and the hyperbolic disk $\mathbb{H}^{2}$. 

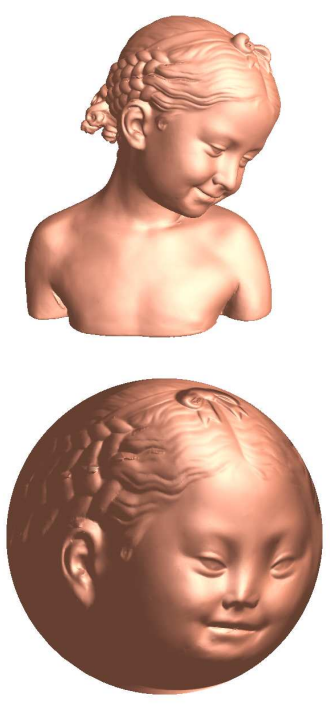

Spherical
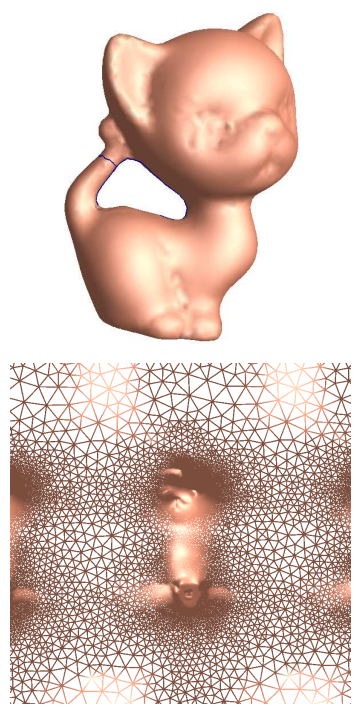

Euclidean
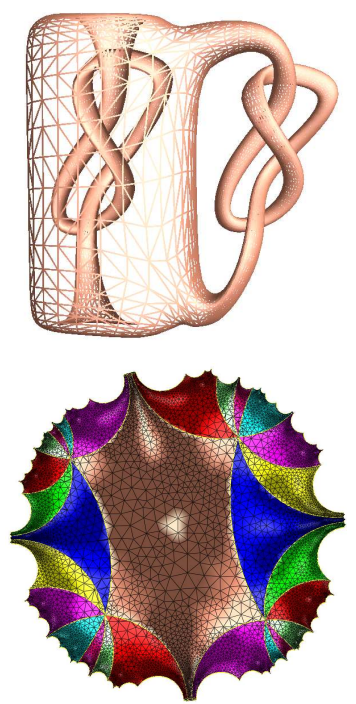

Hyperbolic

FIG. 8. Surface uniformization theorem.

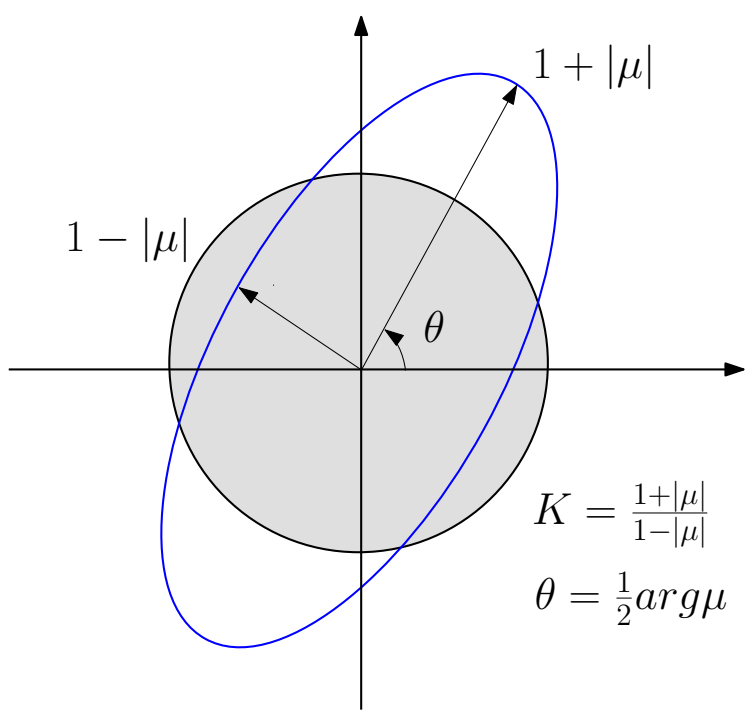

FIG. 9. Illustration of how Beltrami coefficient $\mu$ measures the distortion by a quasi-conformal map that is an ellipse with dilation $K$.

3.6. Quasi-Conformal Maps. A generalization of conformal map is called the quasi-conformal map which is an orientation-preserving homeomorphism between Riemann surfaces with bounded conformality distortion, in the sense that the first order approximation of the quasi-conformal homeomorphism takes small circles to small ellipses of bounded eccentricity. Thus, a conformal homeomorphism that maps a small circle to a small circle can also be regarded as quasi-conformal. 


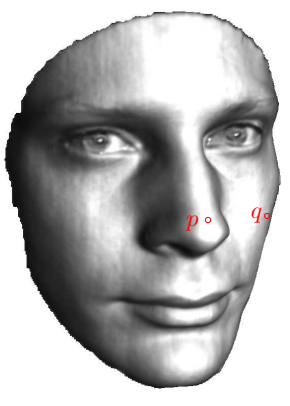

(a) Original face

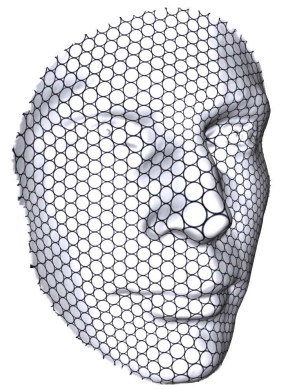

(c) Circle packing induced by (b)

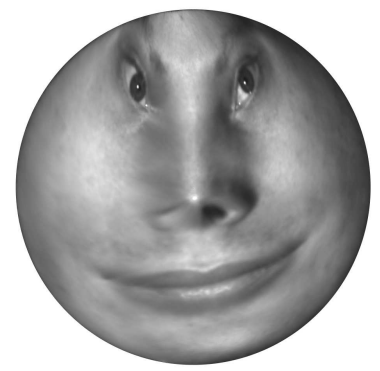

(e) Quasi-conformal mapping

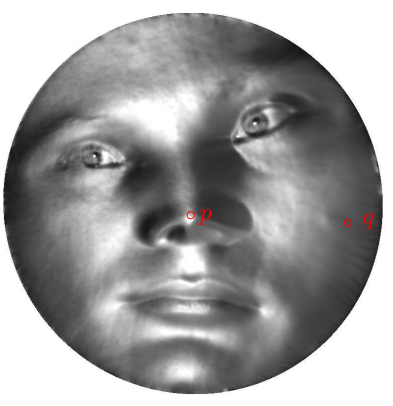

(b) Conformal mapping

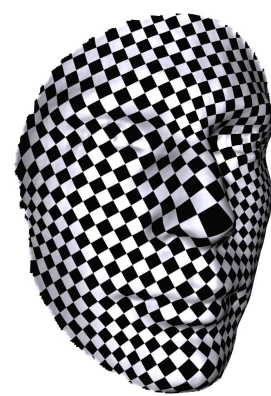

(d) Checker-board texture

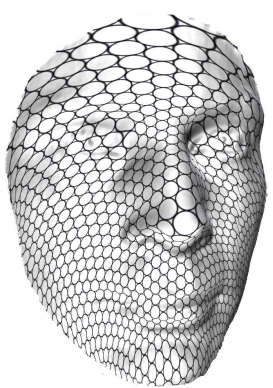

(f) Circle packing induced by (e)

FIG. 10. Conformal and quasi-conformal mappings for a topological disk.

Mathematically, $\phi$ is quasi-conformal provided that it satisfies Beltrami's equation 1 on a local chart for some complex valued Lebesgue measurable $\mu$ satisfying $|\mu|_{\infty}<1$,

$$
\frac{\partial \phi}{\partial \bar{z}}=\mu(z) \frac{\partial \phi}{\partial z}
$$

$\mu$ is called the Beltrami coefficient, which is a measure of conformality. In particular, the map $\phi$ is conformal around a small neighborhood of $p$ when $\mu(p)=0$. In general, $\phi$ maps an infinitesimal circle to a infinitesimal ellipse. From $\mu(p)$, we can determine the angles of the directions of maximal magnification as well as the amount of maximal magnification and maximal shrinking. Specifically, the angle of maximal magnification is $\arg \mu(p) / 2$ with magnifying factor $1+|\mu(p)|$; the angle of maximal shrinking is the 


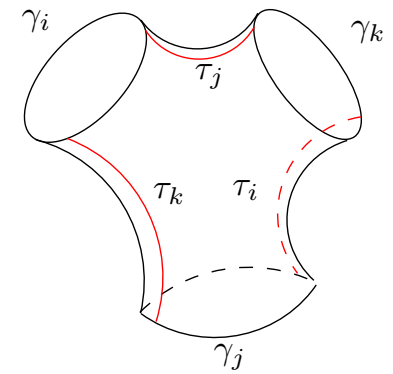

(a) A pair of pants

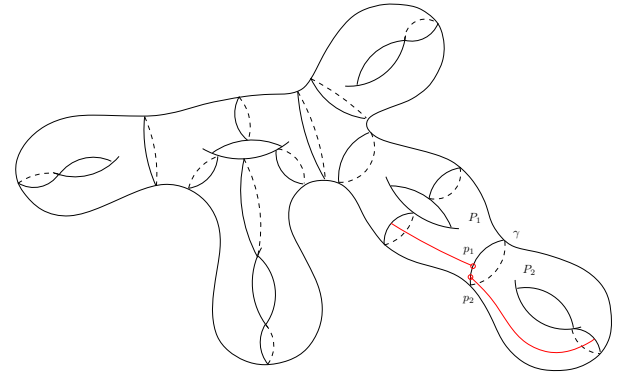

(b) Pants decomposition

FIG. 11. A surface of genus $g$ with a hyperbolic metric is decomposed to $2 g-2$ pairs of pants by cutting along closed geodesics. The twisting angle and length of each cutting loop give the FenchelNielsen coordinates in the shape space.

orthogonal angle $(\arg \mu(p)-\pi) / 2$ with shrinking factor $1-|\mu(p)|$. The distortion or dilation is given by:

$$
K=\frac{1+|\mu(p)|}{1-|\mu(p)|}
$$

Thus, the Beltrami coefficient $\mu$ gives us all the information about the conformality of the map (see Fig. 9).

In terms of the metric tensor, considering the effect of the pullback of the canonical Euclidean metric $\mathbf{g}_{0}$ under $\phi$, the resulting metric is given by:

$$
\left.\phi^{*}\left(\mathbf{g}_{0}\right)=\left|\frac{\partial \phi}{\partial z}\right|^{2} \mid d z+\mu(z) d \bar{z}\right)\left.\right|^{2} .
$$

Figure 10 shows the conformal and quasi-conformal mappings for a topological disk, where the Beltarmi coefficient is set to be $\mu=z$. From the texture mappings in frames (c) and (d), we can see the conformality is kept well around nose tip (circles to circles), while it is changed a lot along boundary area (circles to quasi-ellipses).

3.7. Teichmüller Space. Given a topological surface $S$ with genus $g>1$, all the conformal structures on $S$ form the Moduli space. In general, this space is complicated to compute. Instead, we study its universal covering space Teichmüller space.

Let $S$ be a Riemann surface of genus $g>1$. We mark a set of fundamental group generators $\left\{a_{1}, a_{2}, \cdots, a_{g}, b_{1}, b_{2}, \cdots, b_{g}\right\}$, then $S$ is a marked Riemann surface. Given two marked Riemann surfaces are equivalent, if there is a conformal (bi-holomorphic) map $\phi: S_{1} \rightarrow S_{2}$, such that $\phi$ is isotopic to the identity up to the marking. Each equivalence class is represented as one point in the Teichmüller space $T_{g}$.

The dimension of Teichmüller space is 2 for genus one surfaces, and $6 g-6$ if $g>1$. Because each conformal structure has a unique hyperbolic metric, it is enough to consider surfaces with hyperbolic metrics for computing the Teichüller space.

Assume $S$ is with a hyperbolic metric, then its Fenchel-Nielsen coordinates in $T_{g}$ can be constructed as follows. Given a genus $g$ surface, it can be decomposed to $2 g-2$ 
pairs of pants. Figure 11 illustrates one example. Assume all the cutting loops are geodesics $\left\{\gamma_{1}, \gamma_{2}, \cdots, \gamma_{3 g-3}\right\}$, then each pair of pants is pair of hyperbolic pants.

On each pair of hyperbolic pants $P$ with three boundaries $\gamma_{i}, \gamma_{j}, \gamma_{k}$ there are three shortest paths connecting each pair of boundaries, e.g. $\tau_{i}$ connects $\gamma_{j}, \gamma_{k}$. Then $\tau_{i}, \tau_{j}, \tau_{k}$ separate $S$ to two congruent hyperbolic hexagons with right corner angles.

Suppose two pairs of hyperbolic pants $P_{1}$ and $P_{2}$ are glued together along $\gamma$. The shortest path $\tau_{1}$ on $P_{1}$ intersects $\gamma$ at $p_{1}$, the shortest path $\tau_{2}$ on $P_{2}$ intersects $\gamma$ at $p_{2}$, then the twisting angle on $\gamma$ is given by

$$
\theta=2 \pi \frac{d\left(p_{1}, p_{2}\right)}{|\gamma|}
$$

where $d\left(p_{1}, p_{2}\right)$ is the geodesic distance between $p_{1}$ and $p_{2},|\gamma|$ is the length of $\gamma$. The Fenchel-Nielsen Coordinates are given by the lengths of $\gamma_{k}$ and the twisting angles on $\gamma_{k}, k=1,2, \cdots, 3 g-3$.

\section{Computational Methods.}

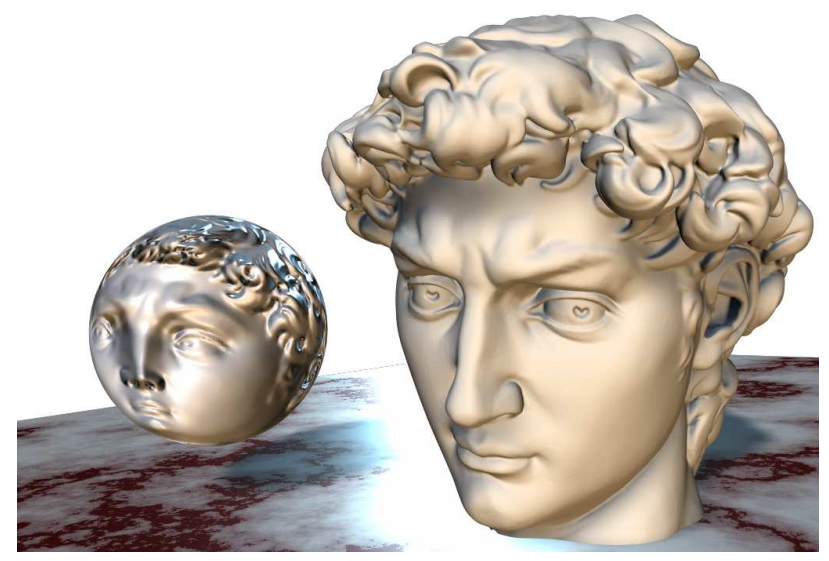

FIG. 12. Harmonic map from a genus zero closed surface to the unit sphere.

4.1. Harmonic Maps. Harmonic maps in $\mathbb{R}^{3}$ can be computed using heat flow method. For example, in case we want to compute a harmonic map from a genus zero closed surface to the unit sphere $\phi: S \rightarrow \mathbb{S}^{2}$. We can initialize the map by the canonical Gauss map, then minimize the harmonic energy by the heat flow. First we compute the Laplacian of the map $\Delta \phi: S \rightarrow \mathbb{R}^{3}$. Then we compute the tangential component of the Laplacian. Suppose $p \in S$, then $\phi(p) \in \mathbb{S}^{2}$.

$$
\Delta^{\perp} \phi(p)=<\Delta^{\perp} \phi(p), \phi(p)>\phi(p) .
$$

The tangential component of the Laplacian is given by

$$
\Delta^{\|} \phi(p)=\Delta \phi(p)-\Delta^{\perp} \phi(p)
$$


The heat flow is defined as

$$
\frac{d \phi(p, t)}{d t}=-\Delta^{\|} \phi(p)
$$

Because the harmonic maps are not unique, they differ by a Möbius transformation on the sphere, special normalization condition needs to be added during the flow. The following is a common condition,

$$
\int_{S} \phi(p) d s=0
$$

For genus zero closed surface, harmonic maps are conformal. Figure 15 shows one example computed using this method.

For genus zero surface with a single boundary, we can convert it to a symmetric closed surface by double covering. Then by mapping the doubled surface to the unit sphere, we can compute the conformal mapping of the original surface. The mapping is not unique. Figure 13 shows that two such conformal mappings differ by a Möbius transformation.
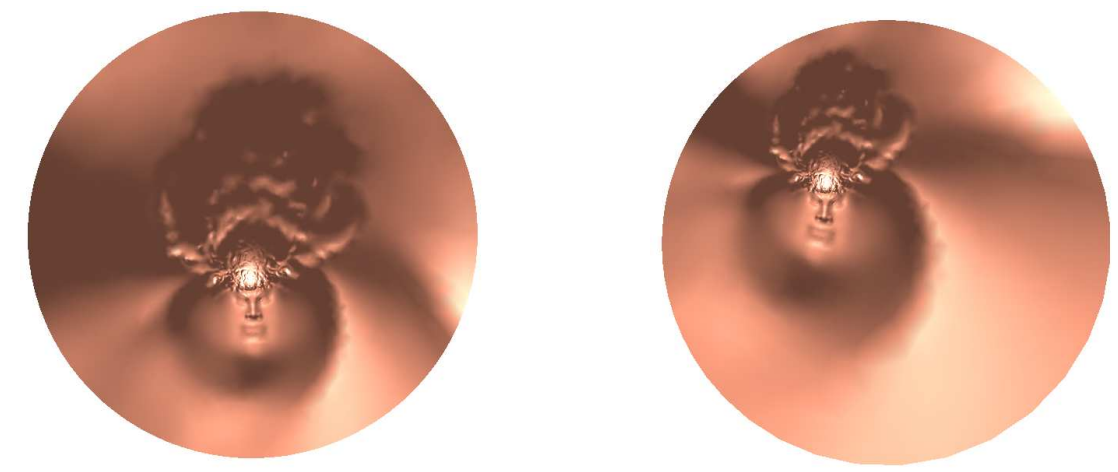

FIG. 13. Conformal mappings from a topological disk to the unit disk differ by a Möbius transformation.
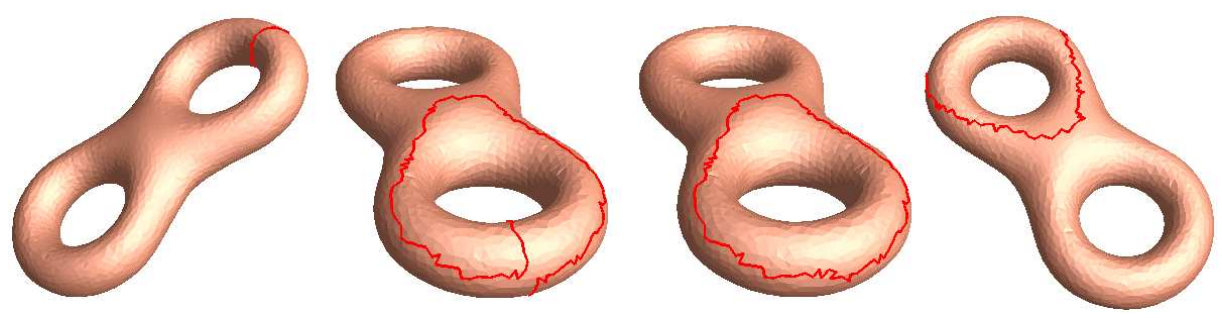

FIG. 14. Computing homology group basis. 


\subsection{Holomorphic Differentials.}

4.2.1. Homology Basis. Given a surface $S$ embedded in $\mathbb{R}^{3}$, we first compute its fundamental group generators. We compute its CW-cell decomposition

$$
S_{0} \subset S_{1} \subset S_{2}=S,
$$

where $S_{k}=S_{k-1} \cup D_{k}^{1} \cup D_{k}^{2} \cdots \cup D_{k}^{n}, D_{k}^{i}$ are $k$-dimensional cells (disks), such that the boundaries of these cells are on $S_{k-1}$,

$$
\partial D_{k}^{i} \subset S_{k-1}
$$

Then the fundamental group of $S_{1}$ has the same generators as the fundamental group of $S$. Then we compute a spanning tree $T$ of $S_{0}$ in $S_{1}$, the complement of $T$ in $S_{1}$ are disconnected 1-cells, denoted as $e_{1}, e_{2}, \cdots e_{k}$, then the union of $T$ and $e_{i}$ has a unique loop $\gamma_{i}$. All such loops $\left\{\gamma_{1}, \gamma_{2}, \cdots, \gamma_{2 g}\right\}$ form a basis for the fundamental group $\pi_{1}(S)$. These loops also form a basis of the first homology basis $H_{1}(S, \mathbb{Z})$. Figure 14 shows the homology group generators of a genus two surface.

4.2.2. Cohomology Basis. Let $\gamma_{k}$ be a base loop for $H_{1}(S, \mathbb{Z})$, then we slice $S$ along $\gamma_{k}$ to get an open surface $S_{k}$, such that the boundary of $S_{k}$ is given by

$$
\partial S_{k}=\gamma_{k}^{+}-\gamma_{k}^{-}
$$

$\gamma_{k}^{+}, \gamma_{k}^{-1}$ are the two boundary loops on $S_{k}$. Then we randomly construct a function $h_{k}: S_{k} \rightarrow \mathbb{R}$, such that

$$
h_{k}(p)=1, \forall p \in \gamma_{k}^{+} ; \quad h_{k}(p)=0, \forall p \in \gamma_{k}^{-} ;
$$

and $h_{k}(p)$ is random for all interior points on $S_{k}$. Then $d h_{k}$ is an exact 1-form on $S_{k}$. Because of the consistency along the boundaries, $d h_{k}$ is also a closed 1-form on $S$. We denote $\tau_{k}$ as $d h_{k}$ on $S$. Then $\left\{\tau_{1}, \tau_{2}, \cdots, \tau_{2 g}\right\}$ form a basis for $H^{1}(S, \mathbb{R})$.
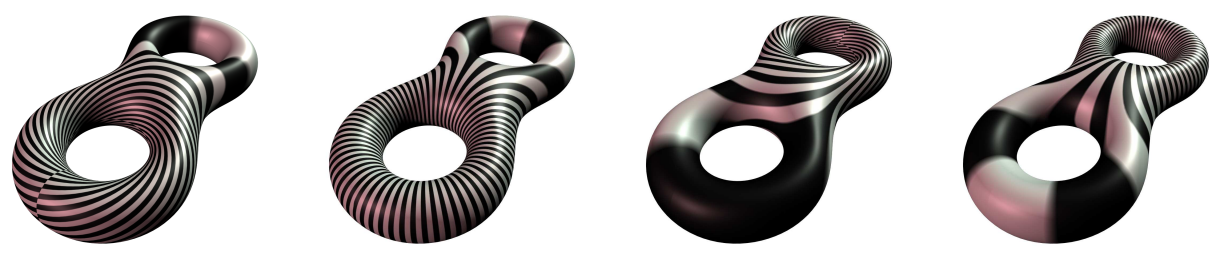

FIG. 15. Computing harmonic 1-form group basis.

4.2.3. Harmonic 1-form Basis. According to Hodge theory, for each closed 1 -form $\tau_{k}$, there exists a 0 -form $g_{k}: S \rightarrow \mathbb{R}$, such that $\tau_{k}+d g_{k}$ is a harmonic 1 -form. The 0 -forma $g_{k}$ can be obtained by solving

$$
d *\left(\tau_{k}+d g_{k}\right)=0
$$


We denote the harmonic 1-form as $\omega_{k}=\tau_{k}+d g_{k}$. Figure 15 shows the harmonic group generators of a genus two surface.
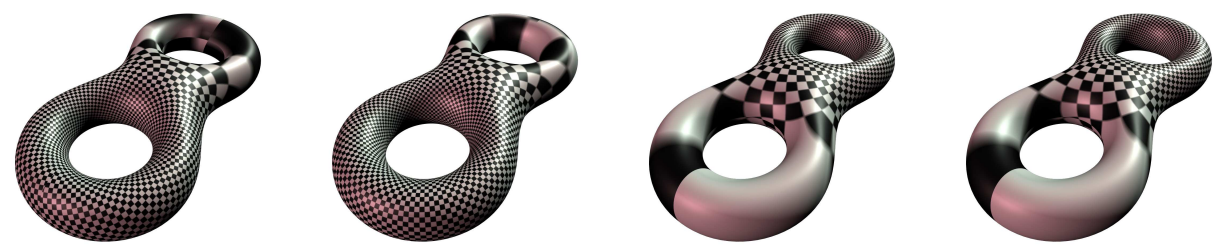

FIG. 16. Computing holomorphic 1-form group basis.

4.2.4. Holomorphic 1-form Basis. Holomorphic 1-form can be constructed by harmonic 1 -form and its conjugate $\omega_{k}+i^{*} \omega_{k}$. Then $\left\{\omega_{1}+i^{*} \omega_{1}, \omega_{2}+i^{*} \omega_{2}, \cdots, \omega_{2 g}+\right.$ $\left.i^{*} \omega_{2 g}\right\}$ form a basis for holomorphic 1-form group. Figure 16 shows the holomorphic 1-form group basis for the genus two surface.

For surfaces with boundaries, we can convert the surface to a symmetric closed surface by the double covering technique.

4.3. Discrete Surface Ricci Flow. In engineering field, smooth surfaces are often approximated by simplicial complexes (triangle meshes). Major concepts, such as metric, curvature, and conformal deformation in the continuous setting can be generalized to the discrete setting. We denote a triangle mesh as $\Sigma$, a vertex set as $V$, an edge set as $E$, and a face set as $F$. $e_{i j}$ represents the edge connecting vertices $v_{i}$ and $v_{j}$, and $f_{i j k}$ denotes the face formed by $v_{i}, v_{j}$, and $v_{k}$.

4.3.1. Background Geometry. In engineering field, it is always assumed that a mesh $\Sigma$ is embedded in the three dimensional Euclidean space $\mathbb{R}^{3}$, and therefore each face is Euclidean. In this case, we say the mesh is with Euclidean background geometry. The angles and edge lengths of each face satisfy the Euclidean cosine law.

Similarly, we can assume that a mesh is embedded in the three dimensional sphere $\mathbb{S}^{3}$ or hyperbolic space $\mathbb{H}^{3}$, then each face is a spherical or a hyperbolic triangle. We say the mesh is with spherical or hyperbolic background geometry. The angles and the edge lengths of each face satisfy the spherical or hyperbolic cosine law.

4.3.2. Discrete Riemannian Metric. A discrete Riemannian metric on a mesh $\Sigma$ is a piecewise constant metric with cone singularities. A metric on a mesh with Euclidean metric is a discrete Euclidean metric with cone singularities. Each vertex is a cone singularity. Similarly, a metric on a mesh with spherical background geometry is a discrete spherical metric with cone singularities; a metric on a mesh with hyperbolic background geometry is a discrete hyperbolic metric with cone singularities. 


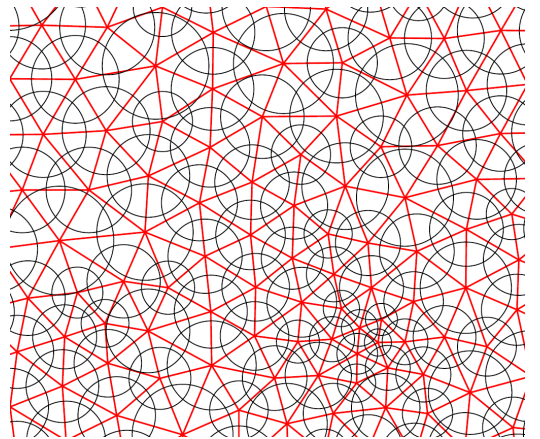

(a)

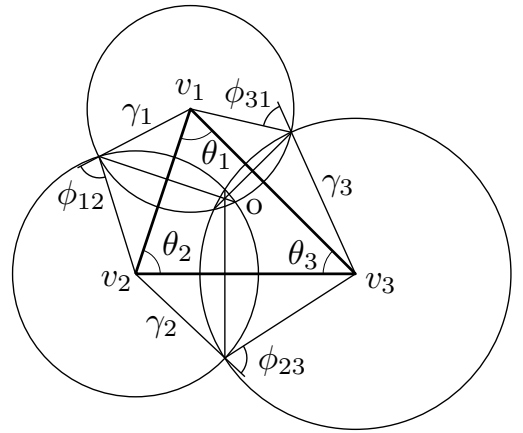

(b)

FIG. 17. Circle Packing Metric. (a) Flat circle packing metric, (b) Circle packing metric on a triangle.

The edge lengths of a mesh $\Sigma$ are sufficient to define a discrete Riemannian metric,

$$
l: E \rightarrow \mathbb{R}^{+},
$$

as long as, for each face $f_{i j k}$, the edge lengths satisfy the triangle inequality: $l_{i j}+l_{j k}>$ $l_{k i}$ for all the three background geometries, and another inequality: $l_{i j}+l_{j k}+l_{k i}<2 \pi$ for spherical geometry.

4.3.3. Discrete Gaussian Curvature. The discrete Gaussian curvature $K_{i}$ on a vertex $v_{i} \in \Sigma$ can be computed from the angle deficit,

$$
K_{i}=\left\{\begin{array}{cc}
2 \pi-\sum_{f_{i j k} \in F} \theta_{i}^{j k}, & v_{i} \notin \partial \Sigma \\
\pi-\sum_{f_{i j k} \in F} \theta_{i}^{j k}, & v_{i} \in \partial \Sigma
\end{array}\right.
$$

where $\theta_{i}^{j k}$ represents the corner angle attached to vertex $v_{i}$ in the face $f_{i j k}$, and $\partial \Sigma$ represents the boundary of the mesh. The discrete Gaussian curvatures are determined by the discrete metrics.

4.3.4. Discrete Gauss-Bonnet Theorem. The Gauss-Bonnet theorem states that the total curvature is a topological invariant. It still holds on meshes as follows.

$$
\sum_{v_{i} \in V} K_{i}+\lambda \sum_{f_{i} \in F} A_{i}=2 \pi \chi(M)
$$

where $A_{i}$ denotes the area of face $f_{i}$, and $\lambda$ represents the constant curvature for the background geometry; +1 for the spherical geometry, 0 for the Euclidean geometry, and -1 for the hyperbolic geometry.

4.3.5. Circle Packing Metric. The concept of the circle packing metric was introduced by Thurston in [46] as shown in Fig. 17. Let $\Gamma$ be a function defined on the vertices, $\Gamma: V \rightarrow \mathbb{R}^{+}$, which assigns a radius $\gamma_{i}$ to the vertex $v_{i}$. Similarly, let $\Phi$ be a function defined on the edges, $\Phi: E \rightarrow\left[0, \frac{\pi}{2}\right]$, which assigns an acute angle 
$\Phi\left(e_{i j}\right)$ to each edge $e_{i j}$ and is called a weight function on the edges. Geometrically, $\Phi\left(e_{i j}\right)$ is the intersection angle of two circles centered at $v_{i}$ and $v_{j}$. The pair of vertex radius function and edge weight function on a mesh $\Sigma,(\Gamma, \Phi)$, is called a circle packing metric of $\Sigma$. Two circle packing metrics $\left(\Gamma_{1}, \Phi_{1}\right)$ and $\left(\Gamma_{2}, \Phi_{2}\right)$ on the same mesh are conformally equivalent if $\Phi_{1} \equiv \Phi_{2}$.

4.3.6. Admissible Curvature Space. A mesh $\Sigma$ with edge weight $\Phi$ is called a weighted mesh, which is denoted as $(\Sigma, \Phi)$. In the following, we want to clarify the spaces of all possible circle packing metrics and all possible curvatures of a weighted mesh.

Let the vertex set be $V=\left\{v_{1}, v_{2}, \cdots, v_{n}\right\}$, and the radii be $\Gamma=\left\{\gamma_{1}, \gamma_{2}, \cdots, \gamma_{n}\right\}$. Let $u_{i}$ be

$$
u_{i}= \begin{cases}\log \gamma_{i} & \mathbb{E}^{2} \\ \log \tanh \frac{\gamma_{i}}{2} & \mathbb{H}^{2} \\ \log \tan \frac{\gamma_{i}}{2} & \mathbb{S}^{2}\end{cases}
$$

where $\mathbb{E}^{2}, \mathbb{H}^{2}$, and $\mathbb{S}^{2}$ indicate the background geometry of the mesh. We represent a circle packing metric on $(\Sigma, \Phi)$ by a vector $\mathbf{u}=\left(u_{1}, u_{2}, \cdots, u_{n}\right)^{T}$. Similarly, we represent the Gaussian curvatures at mesh vertices by the curvature vector $\mathbf{k}=\left(K_{1}, K_{2}, \cdots, K_{n}\right)^{T}$. All the possible $\mathbf{u}$ 's form the admissible metric space, and all the possible k's form the admissible curvature space.

According to the Gauss-Bonnet theory (see Eqn. 6), the total curvature must be $2 \pi \chi(\Sigma)$, and therefore the curvature space is $n-1$ dimensional. We add one linear constraint to the metric vector $\mathbf{u}, \sum u_{i}=0$, for the normalized metric. As a result, the metric space is also $n-1$ dimensional. If all the intersection angles are acute, then the edge lengths induced by a circle packing satisfy the triangle inequality. There is no further constraint on $\mathbf{u}$. Therefore, the admissible metric space is simply $\mathbb{R}^{n-1}$.

A curvature vector $\mathbf{k}$ is admissible if there exists a metric vector $\mathbf{u}$, which induces k. The admissible curvature space of a weighted mesh $(\Sigma, \Phi)$ is a convex polytope, specified by the following theorem. The detailed proof can be found in [32].

The admissible curvature space for weighted meshes with hyperbolic or spherical background geometries is more complicated. We refer the readers to [47] for detailed discussion.

4.3.7. Ricci Flow. Suppose $(\Sigma, \Phi)$ is a weighted mesh with an initial circle packing metric. The discrete Ricci flow is defined as follows.

$$
\frac{d u_{i}(t)}{d t}=\left(\bar{K}_{i}-K_{i}\right),
$$

where $\overline{\mathbf{k}}=\left(\bar{K}_{1}, \bar{K}_{2}, \cdots, \bar{K}_{n}\right)^{T}$ is the user defined target curvature. The discrete Ricci flow has exactly the same form as the smooth Ricci flow, which deforms the circle packing metric according to the Gaussian curvature, as in Eqn. 8. 
The discrete Ricci flow can be formulated in the variational setting, namely, it is a negative gradient flow of a special energy form. The energy is given by

$$
f(\mathbf{u})=\int_{\mathbf{u}_{\mathbf{0}}}^{\mathbf{u}} \sum_{i=1}^{n}\left(\bar{K}_{i}-K_{i}\right) d u_{i},
$$

where $\mathbf{u}_{\mathbf{0}}$ is an arbitrary initial metric. The energy is called the discrete Ricci energy.

Computing the desired metric with user-defined curvature $\overline{\mathbf{k}}$ is equivalent to minimizing the discrete Ricci energy. For Euclidean or hyperbolic cases, the discrete Ricci energy (see Eqn. 9) was first proved to be strictly convex in the seminal work of Colin de Verdiere [30] for the $\Phi=0$ case, and was generalized to all cases of $\Phi \leq \pi / 2$ in [32]. The global minimum uniquely exists, corresponding to the metric $\overline{\mathbf{u}}$, which induces $\overline{\mathbf{k}}$. The discrete Ricci flow converges to this global minimum. Although the spherical Ricci energy is not strictly convex, the desired metric $\overline{\mathbf{u}}$ is still a critical point of the energy.

The energy can be optimized using Newton's method. As shown in Fig. 17 (b), for each face $\left[v_{i}, v_{j}, v_{k}\right]$ there exists a unique circle orthogonal to all three circles at the vertices, whose center is $o$. The distance from the center to edge $\left[v_{i}, v_{j}\right]$ is denoted as $d_{i j}^{k}$. The weight for an edge $\left[v_{i}, v_{j}\right]$ adjacent to $\left[v_{i}, v_{j}, v_{k}\right]$ and $\left[v_{j}, v_{i}, v_{l}\right]$ is defined as

$$
\mu_{i j}=d_{i j}^{k}+d_{i j}^{l}
$$

The Hessian matrix $H=\left(h_{i j}\right)$ is given by the discrete Laplace form

$$
h_{i j}=\left\{\begin{aligned}
0, & {\left[v_{i}, v_{j}\right] \notin E } \\
d_{i j}, & i \neq j \\
\sum_{k} d_{i k}, & i=j
\end{aligned}\right.
$$
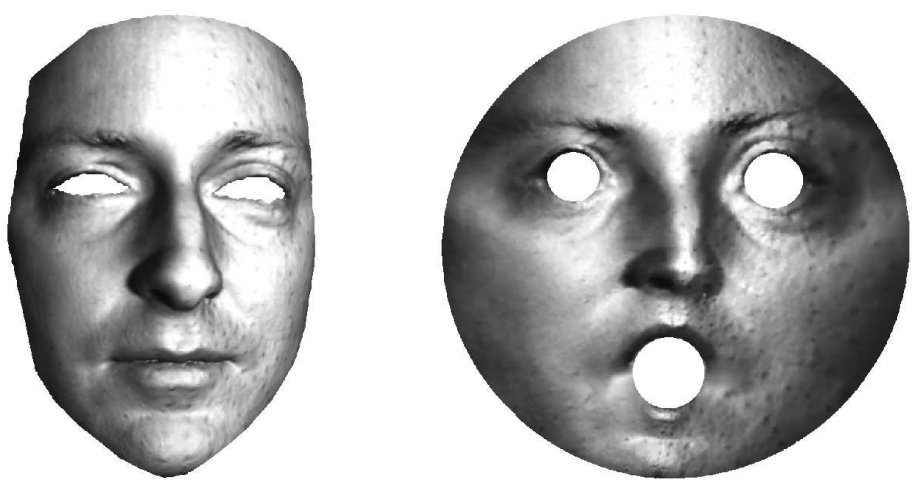

FIG. 18. Euclidean Ricci flow method to compute a conformal mapping for a multiply connected domain. 

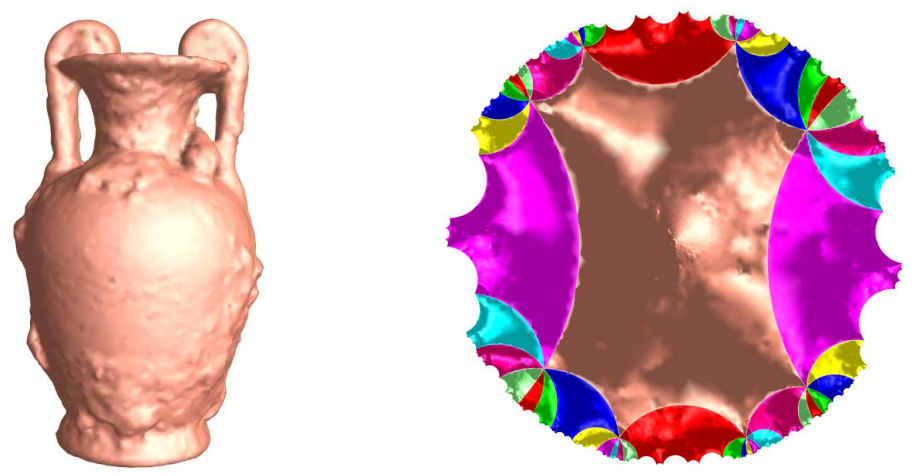

FIG. 19. Hyperbolic Ricci flow method to compute a conformal mapping for a genus two surface.

Figure 18 shows one example for computing the conformal mapping of a multiply connected domain onto the plane. Figure 19 shows the result for computing the uniformization hyperbolic metric for a genus two surface. The universal covering space of the surface with the uniformization metric is isometrically embedded in the Poicaré disk.

4.4. Discrete Surface Yamabe Flow. For smooth surfaces, the Ricci flow and Yamabe flow are equivalent. Using the symbols in the previous discussion, let $\Sigma$ be a triangle mesh embedded in $\mathbb{R}^{3}$. Let $e_{i j}$ be an edge with end vertices $v_{i}$ and $v_{j} . d_{i j}$ is the edge length of $e_{i j}$ induced by the Euclidean metric of $\mathbb{R}^{3}$. A function defined on the vertices $\mathbf{u}: V \rightarrow \mathbb{R}$ is the discrete conformal factor. The edge length $l_{i j}$ is defined as

$$
l_{i j}=e^{u_{i}+u_{j}} d_{i j}
$$

Let $K_{i}$ and $\bar{K}_{i}$ denote the current vertex curvature and the target vertex curvature respectively. The discrete Yamabe flow is defined as

$$
\frac{d u_{i}(t)}{d t}=\bar{K}_{i}-K_{i}
$$

with initial condition $u_{i}(0)=0$. The convergence of Yamabe flow is proven in [42]. Furthermore, Yamabe flow is the gradient flow of the following Yamabe energy, let $\mathbf{u}=\left(u_{1}, u_{2}, \cdots, u_{n}\right), n$ is the total number of vertices,

$$
f(\mathbf{u})=\int_{\mathbf{u}_{0}}^{\mathbf{u}} \sum_{i}^{n}\left(\bar{K}_{i}-K_{i}\right) d u_{i} .
$$

The Yamabe energy is well defined and convex. The Hessian matrix can be easily constructed as follows. Suppose faces $\left[v_{i}, v_{j}, v_{k}\right]$ and $\left[v_{j}, v_{i}, v_{l}\right]$ are adjacent to the edge $e_{i j}$, define the weight of the edge $e_{i j}$ as

$$
w_{i j}=\cot \theta_{k}+\cot \theta_{l},
$$


where $\theta_{k}$ is the angle at $v_{k}$ in $f_{i j k}, \theta_{l}$ is the angle at $v_{l}$ in face $f_{j i l}$. If the edge is on the boundary, and only attaches to $f_{i j k}$, then

$$
\mu_{i j}=\cot \theta_{k}
$$

It can be shown by direct computation, the differential relation between the curvature and the conformal factor is

$$
d K_{i}=\sum_{j} \mu_{i j}\left(d u_{i}-d u_{j}\right) .
$$

So the Hessian matrix of the yamabe energy $H=\left(h_{i j}\right)$ is given by

$$
h_{i j}= \begin{cases}0 & ,\left[v_{i}, v_{j}\right] \notin E \\ \mu_{i j} & , i \neq j \\ -\sum_{k} \mu_{i k} & , i=j\end{cases}
$$

The Hessian matrix is positive definite on the linear subspace $\sum_{i} u_{i}=0$. By using the Hessian matrix (see Eqn. 15), the Yamabe energy (see Eqn. 12) can be optimized effectively. But the major difficulty is that the admissible metric space $\Omega(\mathbf{u})$ for a mesh with fixed connectivity is not convex,

$$
\Omega(\mathbf{u})=\left\{\mathbf{u} \mid \forall f_{i j k} \in M, l_{i j}+l_{j l}>l_{l i}\right\}
$$

Therefore, during the optimization process using Newton's method, we need to ensure that the metric $\mathbf{u}$ is in the admissible metric space $\Omega(u)$ at each step. If a degenerated triangle $f_{i j k}$ is detected, then we swap the longest edge of it. For example, if $\theta_{k}$ exceeds $\pi$, then we swap edge $e_{i j}$ as shown in Fig. 20. The major difficulty for

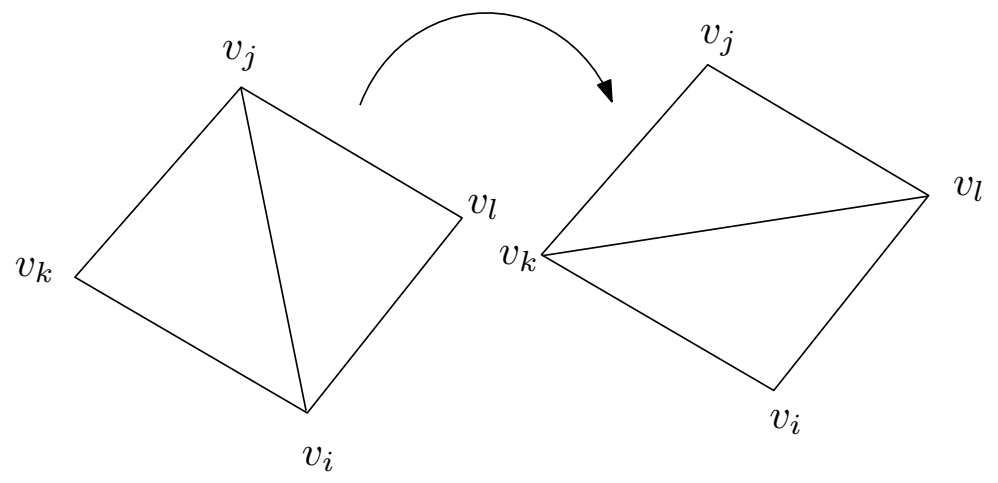

FIG. 20. Edge swap.

the discrete Ricci flow is to find a good initial circle packing with all acute edge intersection angles. This problem does not exist for discrete Yamabe flow. Therefore, yamabe flow in general produces better conformality in practice. Figure 21 shows the conformal brain mapping result using Yamabe flow method. 

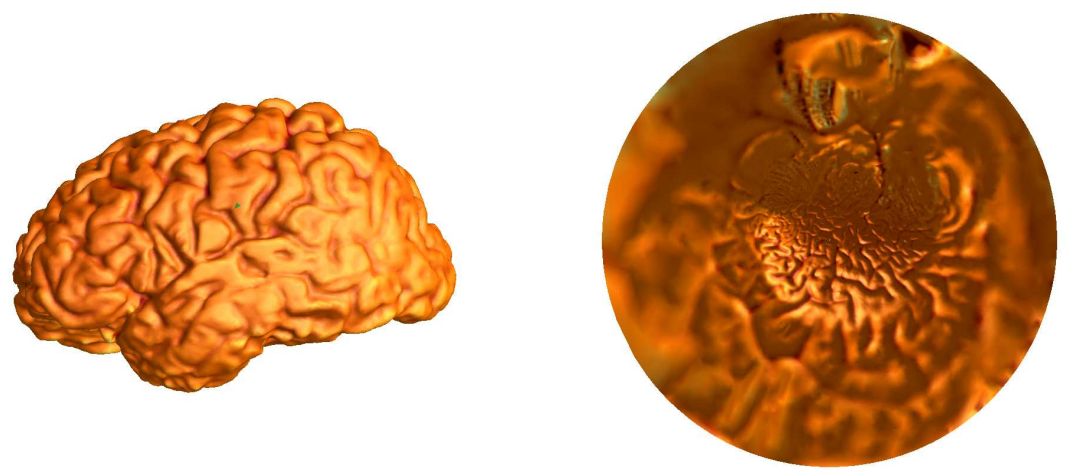

FIG. 21. Conformal brain mapping using Yamabe flow method.

4.5. Quasi-Conformal Mapping by Solving Beltrami Equations. Given a surface $S$, with a conformal structure, also given a measurable complex value function defined on the surface $\mu: S \rightarrow \mathbb{C}$, we want to find a quasi-conformal map $\phi: S \rightarrow \mathbb{C}$, such that $\phi$ satisfies the Beltrami equation:

$$
\frac{\partial \phi}{\partial \bar{z}}=\mu \frac{\partial \phi}{\partial z}
$$

First we construct a conformal mapping $\phi_{1}: S \rightarrow \mathbb{D}_{1}$, where $\mathbb{D}_{1}$ is a planar domain on C. Let $\mathbf{g}_{0}$ be the canonical Euclidean metric on the plane $\mathbf{g}_{0}=d z d \bar{z}$. $\phi_{1}$ is a conformal map from metric surface $(S, \mathbf{g})$ to a metric surface $\left(\mathbb{D}_{1}, \mathbf{g}_{0}\right)$. Then we construct a new metric on $\left(\mathbb{D}_{1}, \mathbf{g}_{0}\right)$, such that

$$
\mathbf{g}_{1}=|d z+\mu d \bar{z}|^{2} .
$$

Then we construct another conformal map $\phi_{2}:\left(\mathbb{D}_{1}, \mathbf{g}_{1}\right) \rightarrow\left(\mathbb{D}_{2}, \mathbf{g}_{0}\right)$. Then the composition

$$
\phi=\phi_{2} \circ \phi_{1}:(S, \mathbf{g}) \rightarrow\left(\mathbb{D}_{2}, \mathbf{g}_{0}\right)
$$

is the desired quasi-conformal mapping. Figure 22 illustrates quasi-conformal mappings for a doubly connected domain with different Beltrami coefficients. For details, we refer readers to [48].

5. Applications. The conformal geometric methods have been broadly applied in many engineering fields. In the following, we briefly introduce their major applications in graphics, vision, geometric modeling and medical imaging.

\subsection{Computing Conformal Mappings.}

5.1.1. Doubly Connected Domain. As shown in Fig. 23, a doubly connected domain is conformally mapped to a canonical planar annulus. The mapping can be constructed using holomorphic 1-form. The holomorphic 1-form group on the surface 

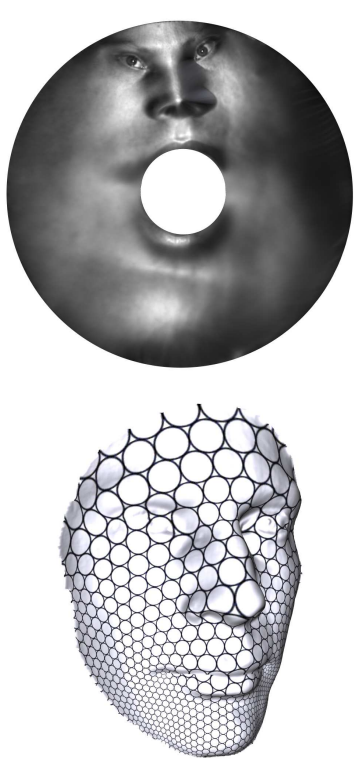

(a) $\mu=0$
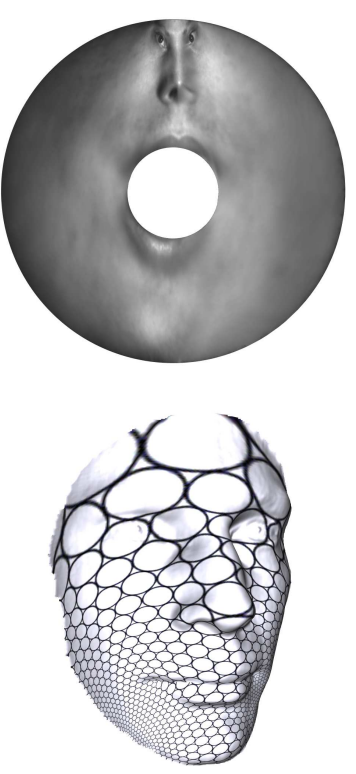

(b) $\mu=0.25+0.0 i$
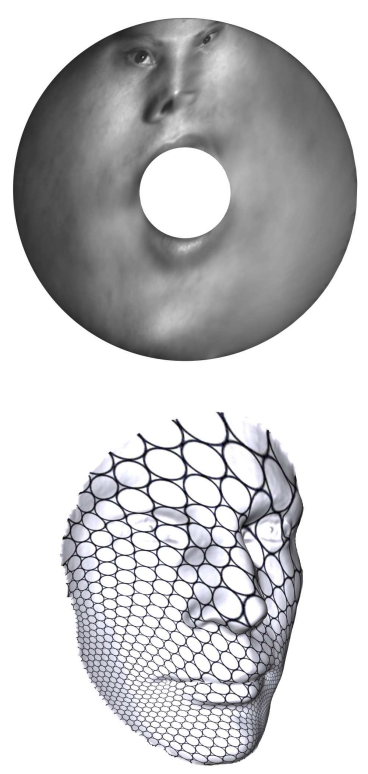

(c) $\mu=0.0+0.25 i$

FIG. 22. Quasi-Conformal mapping for doubly connected domain.

is of one dimension. Let $\omega$ be the generator. Suppose $\partial S=\gamma_{1}-\gamma_{2}$, then we can find a constant $c$, such that

$$
\operatorname{Im}\left(\int_{\gamma_{1}} c \omega\right)=2 \pi i, \quad \operatorname{Im}\left(\int_{\gamma_{2}} c \omega\right)=-2 \pi i .
$$

Then we choose one point $p \in S$, for any point $q \in S$, find a path $\gamma \subset S$, define

$$
\phi(q): q \rightarrow \exp \int_{\gamma} c \omega,
$$

maps the surface to the planar annulus.

The conformal mapping can be constructed using either Ricci flow or Yamabe flow. We set the target curvature equals to zero for both interior vertices and boundary vertices. Then by using curvature flow, we get a flat metric $\overline{\mathbf{g}}$. We isometrically embed the universal covering space of $S$ with $\overline{\mathbf{g}}$ onto the plane, then make each fundamental polygon a rectangle, with the period equal to $2 \pi$, then by using exponential map, we can map each fundamental polygon to a planar annulus.

5.1.2. Simply Connected Domains. The Riemann mapping for a topological disk to the canonical planar unit disk can be constructed in the following way (as shown in Fig. 24). We choose a interior point $p$, and a point $q$ on the boundary. Then remove a small neighborhood around $p$, then we get an annulus $\tilde{S}$. Using holomorphic 1-form or curvature flow method, we map $\tilde{S}$ to a planar unit annulus, the two boundary circles are concentric. Then by a rotation, we can map $q$ to the 

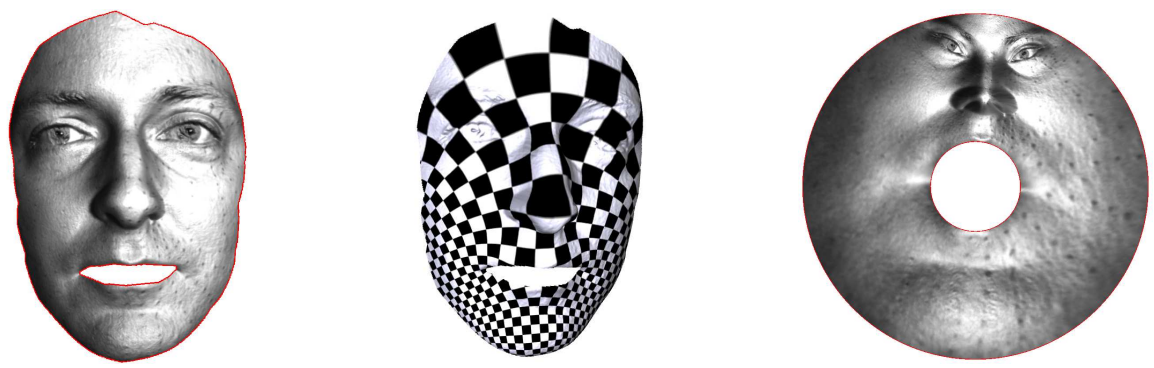

FIG. 23. Conformal mapping for a doubly connected domain.
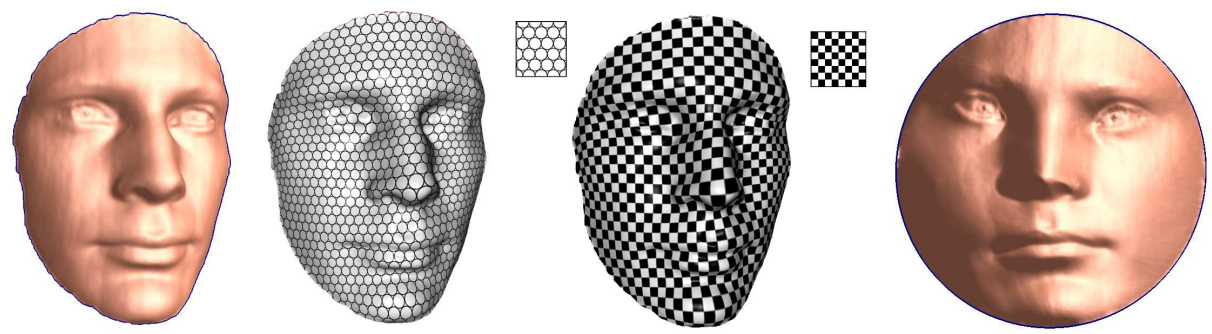

FIG. 24. Conformal mapping for a simply connected domain.

point 1 . We denote the mapping as $\tilde{\phi}: \tilde{S} \rightarrow A$. By shrinking the neighborhood of $p, \tilde{\phi}$ converges to the unique Riemann mapping $\phi: S \rightarrow \mathbb{D}$, such that $\phi(p)=0, \phi(q)=1$.

5.1.3. Multiply Connected Domains. A multiply connected domain as shown in Fig. 25 can be conformally mapped to the unit disk with circular holes. Let the boundary of the surface

$$
\partial S=\gamma_{0}-\gamma_{1}-\gamma_{2}-\cdots-\gamma_{n}
$$

where $\gamma_{0}$ is the exterior boundary. The computation procedure is as follows: choose $\gamma_{k}, 1<k<n$, fill $\gamma_{i}$ 's with disks, where $i \neq 0, i \neq k$, denote the resulting surface as $S_{k}$. Then we find a conformal mapping $\phi_{k}: S_{k} \rightarrow \mathbb{D}_{k}$. Then we remove all filled disks from $D_{k}$. Choose different inner boundary, repeat the above procedure. The inner holes become rounder and rounder. With appropriate normalization condition, (the conformal mapping is not unique, different mappings differ by a Möbius transformation), the mappings converge with very fast rate.

5.1.4. Comparison. Curvature flow method is non-linear and requires higher quality of triangulation, therefore it is less robust and less efficient comparing to the holomorphic 1-form method.

5.1.5. Quasi-Conformal Maps. All the above algorithms can be directly generalized to compute quasi-conformal mappings by solving Beltrami equations. 

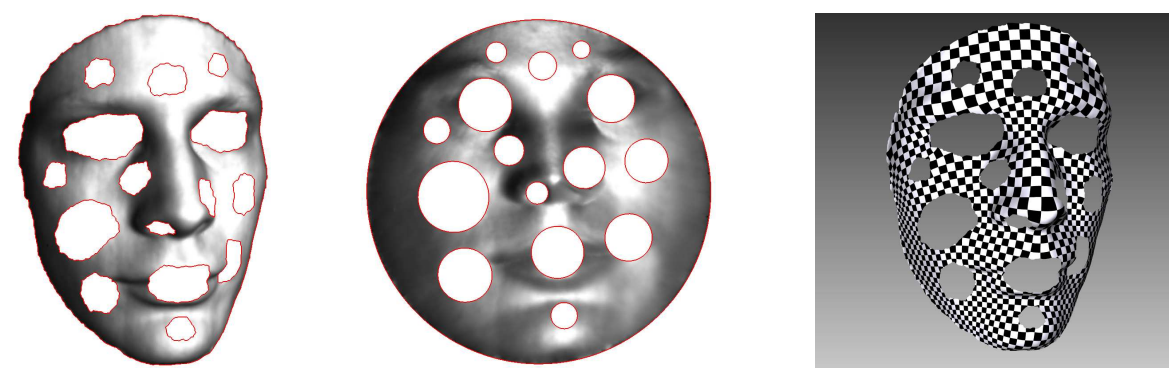

FIG. 25. Conformal mapping for a multiply connected domain.

5.2. Geometric Modeling. One of the most fundamental problems in geometric modeling is to systematically generalize conventional Spline schemes from Euclidean domains to manifold domains. Conventional spline schemes are constructed based on Affine invariance. If the manifold has an affine structure, then affine geometry can be defined on the manifold, therefore, conventional splines can be directly defined on the manifold. Due to the topological obstruction, general manifolds don't have affine structures, but by removing several singularities, general surfaces can admit affine structures.

Affine structures can be explicitly constructed using the methods introduced above. For example, we can concentrate all the curvatures at the prescribed singularity positions, and set the target curvatures to be zeros everywhere else. Then we use curvature flow to compute a flat metric with cone singularities from the prescribed curvature. The flat metric induces an atlas on the punctured surface (with singularities removed), such that all the transition functions are rigid motions on the plane. Another approach is to use holomorphic 1-forms, a holomorphic 1-form induces a flat metric with cone singularities at the zeros, where the curvatures are $-2 k \pi$. Figure 26 shows the manifold splines constructed using the curvature flow method.

5.3. Medical Imaging. Conformal geometry has been applied for manifold fields in medical imaging. For example, in brain imaging field, it is crucial to register different brain cortex surfaces. Because brain surfaces are highly convoluted, and different person has different anatomic structures, it is quite challenging to find good matching between cortex surfaces. Figure 27 illustrates one solution by mapping brains to the unit sphere in a canonical way. Then by finding an automorphism, the registration between surfaces can be easily established.

In virtual colonoscopy, the colon surface is reconstructed from CT images. By using conformal geometric method, one can flatten the whole colon surface onto a planar rectangle. Then polyps and other abnormalities can be found efficiently on the planar image. Figure 28 shows an example for virtual colon flattening based on conformal mapping. 

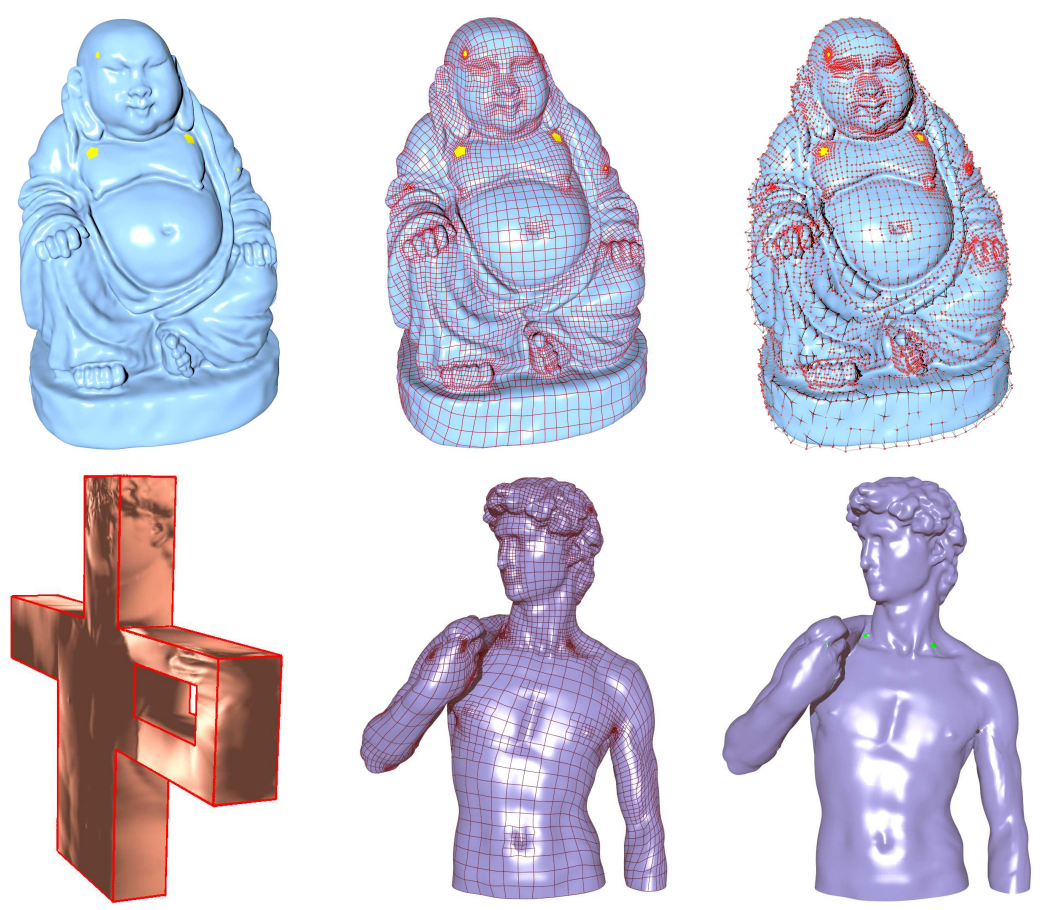

FIG. 26. Manifold splines. The yellow points are extraordinary points.
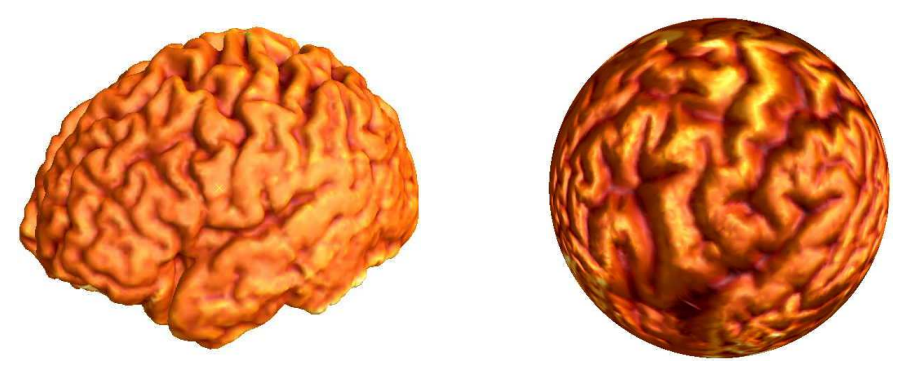

FIG. 27. Brain spherical conformal mapping.

5.4. Vision. Surface matching is a fundamental problem in computer vision. The main framework of surface matching can be formulated in the commutative diagram in Fig. 29.

$S_{1}, S_{2}$ are two given surfaces, $f: S_{1} \rightarrow S_{2}$ is the desired matching. We compute $\phi_{i}: S_{i} \rightarrow D_{i}$ which maps $S_{i}$ conformally onto the canonical domain $D_{i} . D_{1}$ and $D_{2}$ can also be surfaces other than simple planar domains. The topology and the curvature of $D_{1}$ and $D_{2}$ incorporate the major feature information of the original surfaces $S_{1}$ and $S_{2}$. We construct a diffeomorphism map $\bar{f}: D_{1} \rightarrow D_{2}$. If there are certain feature constraints, they can be incorporated in $\bar{f}$. The final map $\phi$ is induced by $f=\phi_{2} \circ \bar{f} \circ \phi_{1}^{-1}$.

Figure 30 shows one example of surface matching among a human face with dif- 

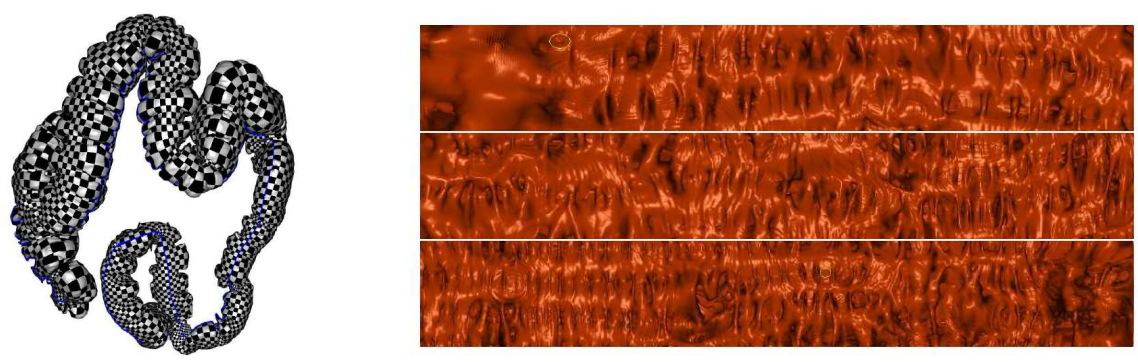

FIG. 28. Colon conformal flattening.

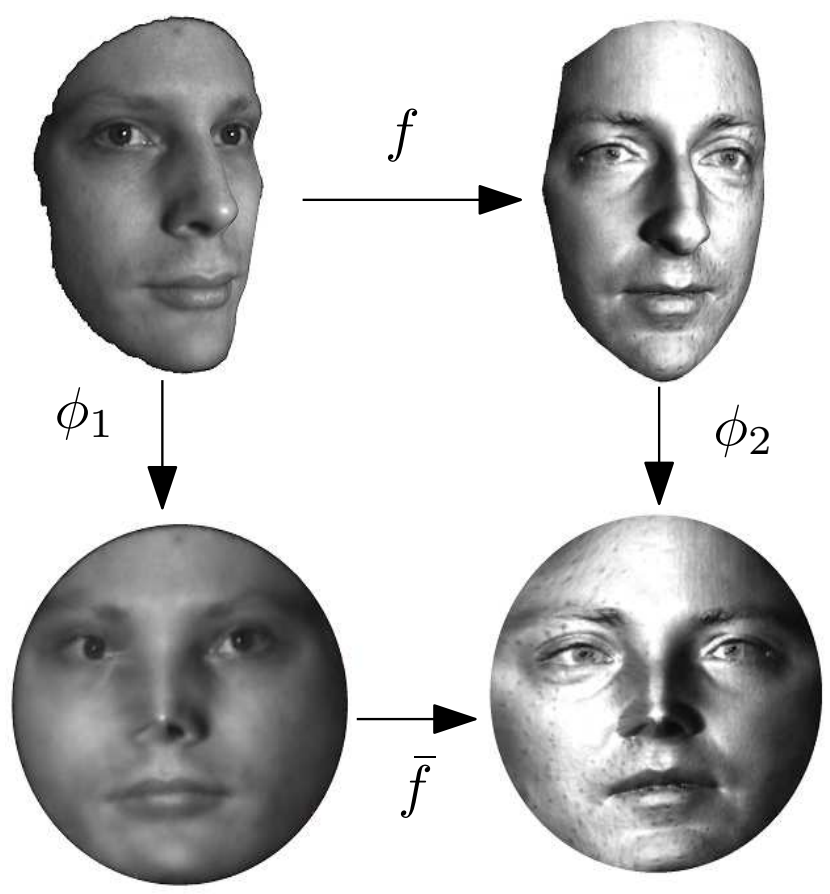

FIG. 29. Surface matching framework.

ferent expressions. The first row shows the surfaces in $\mathbb{R}^{3}$. The second row illustrates the matching results using consistent texture mapping. The intermediate conformal slit mappings are shown in the third row. For details, we refer readers to [22]. Conformal geometric invariants can also be applied for shape analysis and recognition. Details can be found in [49].

Teichmüller theory can be applied for surface classification. By using Ricci curvature flow, we can compute the hyperbolic uniformization metric. Then we compute the pants decomposition using geodesics and compute the Fenchel-Nielsen coordinates. In Fig. 31, a set of canonical fundamental group basis is computed (a). Then a fundamental domain is isometrically mapped to the Poincaré disk with the uniformization 

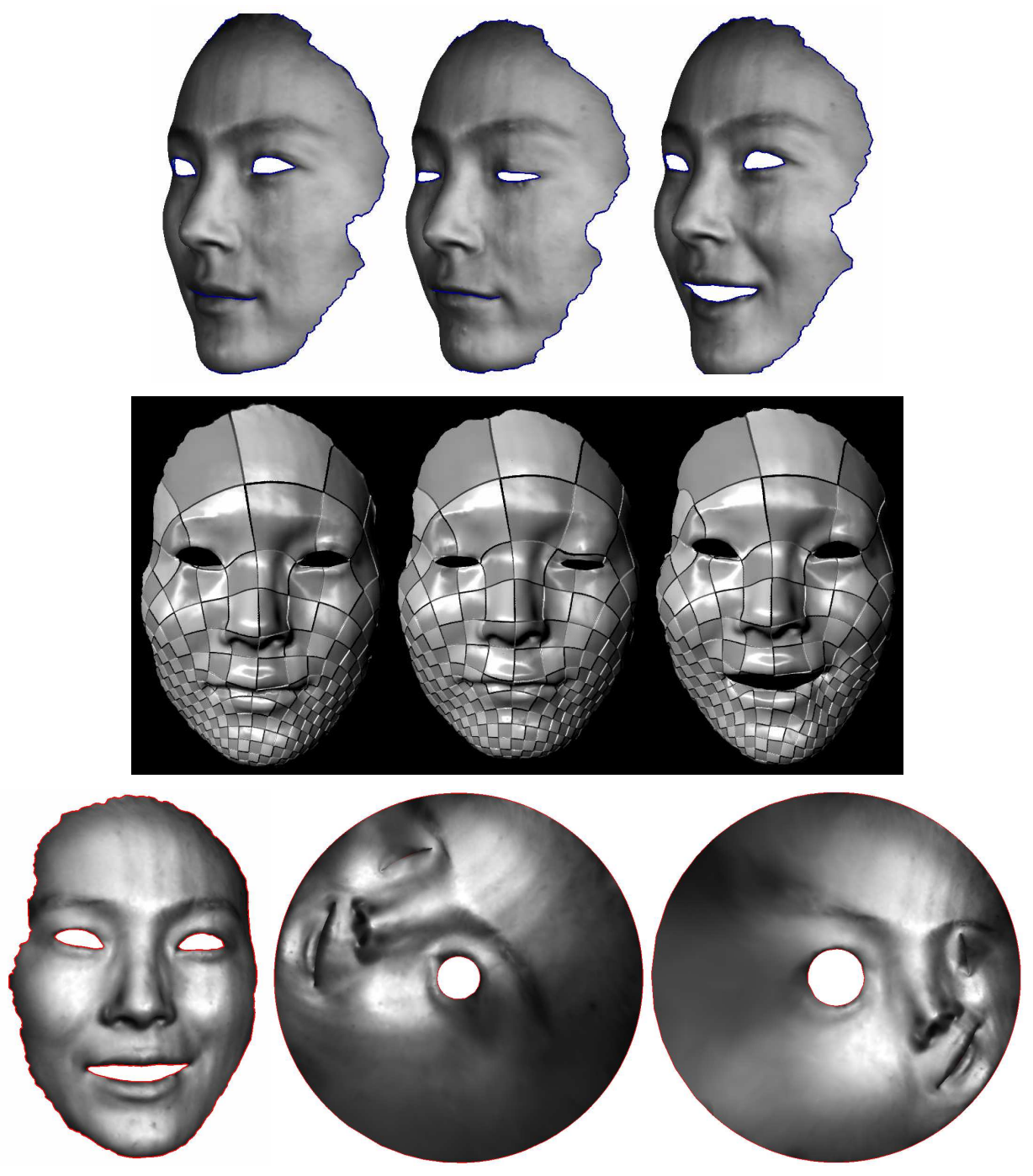

FIG. 30. Matching among faces with different expressions.

metric (b). By using Fuchsian transformation, the fundamental domain is transferred (c) and a finite portion of the universal covering space is constructed in (d). Figure 32 shows the pipeline for computing the Teichmüller coordinates. The geodesics on the hyperbolic disk is found in (a), the surface is decomposed by geodesics (b). The shortest geodesics between two boundaries of each pair of hyperbolic pants are computed in (c),(d) and (e). The twisting angle is computed in (f). Details can be found in [50].

5.5. Graphics. Conformal geometric methods have broad applications in computer graphics. Isothermal coordinates are natural for global surface parameterization purpose. Because conformal mapping doesn't distort the local shapes, it is desirable 


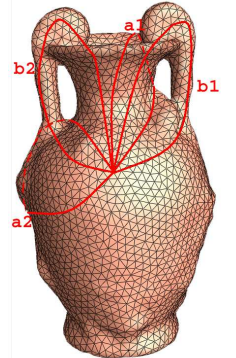

(a)

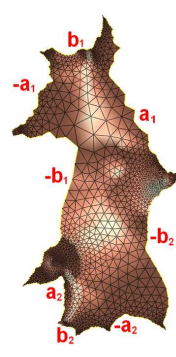

(b)

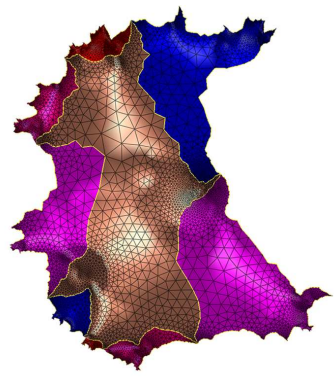

(c)

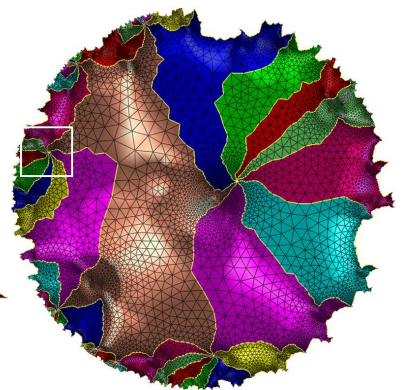

(d)

FIG. 31. Computing finite portion of the universal covering space on the hyperbolic space.

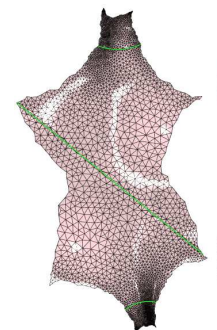

(a)

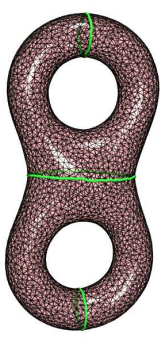

(b)

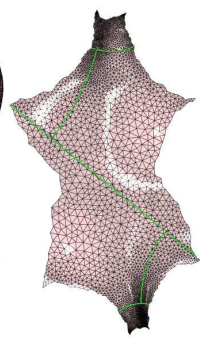

(c)

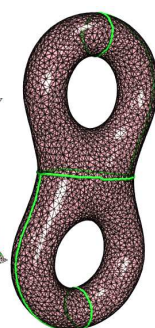

(d)

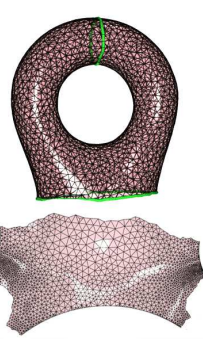

(e)

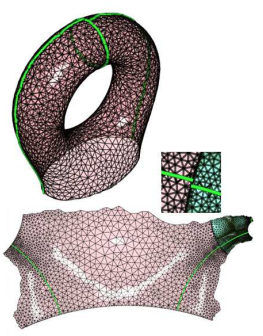

(f)

FIG. 32. Computing the Fenchel-Nielsen coordinates in the Teichmüller space for a genus two surface.

for texture mapping. Figure 33 shows one example of using the holomorphic 1-form for texture mapping.
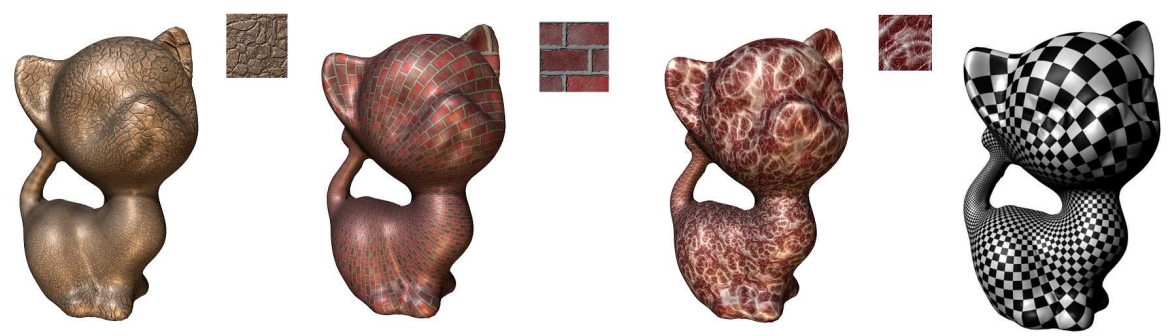

FIG. 33. Global conformal surface parameterization using holomorphic 1-form.

Special flat metrics are valuable for designing vector fields on surfaces, which plays important role for non-photo-realistic rendering and special art form design. Figure 34 shows the examples for vector fields design on surfaces using the curvature flow method.

6. Conclusion. In this work, we briefly summarize the recent developments in computational conformal geometry. There are two major approaches, holomorphic 

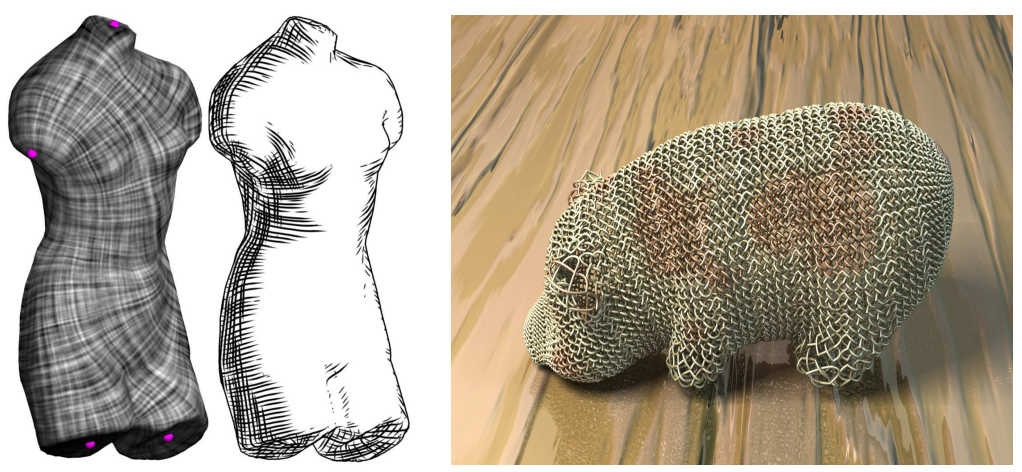

FIG. 34. Vector field design using special flat metrics.

differentials and curvature flow. Holomorphic differential method is a linear method, which is more efficient and robust to triangulations with lower quality. Curvature flow method is nonlinear and requires higher quality triangulations, but more flexible.

There are many future directions in both theories and algorithms:

1. The theoretic foundations for the current computational methods need to be rigorously laid down. The convergence of the discrete conformal structure to the smooth counter part need to be proven. The convergence rate needs to be estimated.

2. More research focuses on quasi-conformal mappings. Especially the computational method for extremal quasi-conformal mappings and Teichmüller theories.

3. Generalization of the computational methods for discrete 3-manifolds. Especially the curvature flow method for canonical geometric structures of 3manifolds.

4. Novel scanning technology which can measure and capture the Beltrami coefficients from real life.

Acknowledgement. We want to thank our collaborators Paul Thompson, Tony Chan, Arie Kaufman, Hong Qin, Dimitris Samaras, Yalin Wang, Lok Ming Lui and many other colleagues. We want to thank our students. The research has been partially supported by NSF CCF-0448399, DMS-0528363, DMS-0626223, CCF-0830550, and ONR N000140910228.

\section{REFERENCES}

[1] P. Henrici, Applide and Computational Complex Analysis, Discrete Fourier Analysis, Cauchy Integrals, COnstruction of Conformal Maps, Univalent Functions. Wiley-Interscience, 1993, vol. 3 .

[2] — - Applide and Computational Complex Analysis, Power Series Integration Conformal Mapping Location of Zero. Wiley-Interscience, 1988, vol. 1. 
[3] M. S. Floater and K. Hormann, Surface parameterization: a tutorial and survey, in: Advances in Multiresolution for Geometric Modelling. Springer, 2005, pp. 157-186.

[4] V. Kraevoy and A. Sheffer, Cross-parameterization and compatible remeshing of $3 d$ models, ACM Transactions on Graphics, 23:3(2004), pp. 861-869.

[5] U. Pinkall and K. Polthier, Computing discrete minimal surfaces and their conjugates, Experimental Mathematics, 2:1(1993), pp. 15-36.

[6] B. Lévy, S. Petitjean, N. Ray, and J. Maillot, Least squares conformal maps for automatic texture atlas generation, SIGGRAPH 2002, pp. 362-371, 2002.

[7] M. Desbrun, M. Meyer, And P. Alliez, Intrinsic parameterizations of surface meshes, Computer Graphics Forum (Proc. Eurographics 2002), 21:3(2002), pp. 209-218.

[8] M. S. Floater, Mean value coordinates, Computer Aided Geometric Design, 20:1(2003), pp. $19-27$.

[9] C. Gotsman, X. Gu, and A. Sheffer, Fundamentals of spherical parameterization for $3 d$ meshes, ACM Transactions on Graphics, 22:3(2003), pp. 358-363.

[10] X. Gu, Y. Wang, T. F. Chan, P. M. Thompson, and S.-T. Yau, Genus zero surface conformal mapping and its application to brain surface mapping, IEEE Trans. Med. Imaging, 23:8(2004), pp. 949-958.

[11] X. Gu And S.-T. YAU, Global conformal parameterization, in: Symposium on Geometry Processing, 2003, pp. 127-137.

[12] . Mercat, Discrete riemann surfaces and the ising model, Communications in Mathematical Physics, 218:1(2004), pp. 177-216.

[13] A. N. Hirani, Discrete exterior calculus, Ph.D. dissertation, California Institute of Technology, 2003.

[14] M. Jin, Y. WANG, S.-T. YAU, AND X. GU, Optimal global conformal surface parameterization, in: IEEE Visualization 2004, 2004, pp. 267-274.

[15] S. J. Gortler, C. Gotsman, And D. Thurston, Discrete one-forms on meshes and applications to $3 D$ mesh parameterization, Computer Aided Geometric Design, 23:2(2005), pp. 83-112.

[16] Y. Tong, P. Alliez, D. Cohen-Steiner, And M. Desbrun, Designing quadrangulations with discrete harmonic forms, in: Symposium on Geometry Processing, 2006, pp. 201-210.

[17] G. Tewari, C. Gotsman, and S. J. Gortler, Meshing genus-1 point clouds using discrete one-forms, Comput. Graph., 30:6(2006), pp. 917-926.

[18] M. Desbrun, Discrete differential forms and applications to surface tiling, in: SCG '06: Proceedings of the twenty-second annual symposium on Computational geometry. ACM, 2006, pp. 40-40.

[19] Y. Tong, P. Alliez, D. Cohen-Steiner, And M. Desbrun, Designing quadrangulations with discrete harmonic forms, in: SGP '06: Proceedings of the fourth Eurographics symposium on Geometry processing, 2006, pp. 201-210.

[20] W. Hong, X. Gu, F. Qiu, M. Jin, And A. E. Kaufman, Conformal virtual colon flattening, in: Symposium on Solid and Physical Modeling, 2006, pp. 85-93.

[21] S. Wang, Y. Wang, M. Jin, X. D. Gu, And D. Samaras, Conformal geometry and its applications on $3 d$ shape matching, recognition, and stitching, IEEE Trans. Pattern Anal. Mach. Intell., 29:7(2007), pp. 1209-1220.

[22] W. Zeng, Y. Zeng, Y. Wang, X. Yin, X. Gu, and D. Samaras, 3d non-rigid surface matching and registration based on holomorphic differentials, in: The 10th European Conference on Computer Vision (ECCV) 2008, 2008, pp. 1-14.

[23] X. Gu, Y. He, And H. Qin, Manifold splines, Graphical Models, 68:3(2006), pp. 237-254.

[24] R. S. Hamilton, Three manifolds with positive Ricci curvature, Journal of Differential Geometry., 17(1982), pp. 255-306.

[25] — - The Ricci flow on surfaces, Mathematics and general relativity (Santa Cruz, CA, 1986), 
Contemp. Math. Amer.Math.Soc. Providence, RI, vol. 71, 1988.

[26] W. P. Thurston, Geometry and Topology of Three-Manifolds. lecture notes at Princeton university, 1980.

[27] Koebe, Kontaktprobleme der Konformen Abbildung, Ber. Sächs. Akad. Wiss. Leipzig, Math.Phys. Kl., vol. 88, pp. 141-164, 1936.

[28] W. P. Thurston, The finite riemann mapping theorem, 1985.

[29] B. Rodin And D. Sullivan, The convergence of circle packings to the riemann mapping, Journal of Differential Geometry, 26:2(1987), pp. 349-360.

[30] C. DE Verdiere Yves, Un principe variationnel pour les empilements de cercles, Invent. Math., 104:3(1991), pp. 655-669.

[31] C. Collins and K. Stephenson, A circle packing algorithm, Computational Geometry: Theory and Applications, 25(2003), pp. 233-256.

[32] B. Chow And F. Luo, Combinatorial Ricci flows on surfaces, Journal Differential Geometry, 63:1(2003), pp. 97-129.

[33] M. Jin, J. Kim, F. Luo, And X. Gu, Discrete surface ricci flow, IEEE Transaction on Visualization and Computer Graphics, 2008.

[34] P. L. Bowers and M. K. Hurdal, Planar conformal mapping of piecewise flat surfaces, in: Visualization and Mathematics III. Berlin: Springer-Verlag, 2003, pp. 3-34.

[35] A. I. Bobenko and B. A. Springborn, Variational principles for circle patterns and koebe's theorem, Transactions of the American Mathematical Society, 356(2004), pp. 659-689.

[36] L. Kharevych, B. Springborn, and P. Schröder, Discrete conformal mappings via circle patterns, ACM Trans. Graph., 25:2(2006), pp. 412-438.

[37] H. Yamabe, The yamabe problem, Osaka Math. J., 12:1(1960), pp. 21-37.

[38] N. S. TRudinger, Remarks concerning the conformal deformation of riemannian structures on compact manifolds, Ann. Scuola Norm. Sup. Pisa, 22:2(1968), pp. 265-274.

[39] T. Aubin, Équations diffréntielles non linéaires et problème de yamabe concernant la courbure scalaire, J. Math. Pures Appl., 55:3(1976), pp. 269-296.

[40] R. Schoen, Conformal deformation of a riemannian metric to constant scalar curvature, J. Differential Geom., 20:2(1984), pp. 479-495.

[41] J. M. Lee And T. H. PARker, The yamabe problem, Bulletin of the American Mathematical Society, 17:1(1987), pp. 37-91.

[42] F. Luo, Combinatorial yamabe flow on surfaces, Commun. Contemp. Math., 6:5(2004), pp. $765-780$.

[43] B. Springborn, P. Schröoder, and U. Pinkall, Conformal equivalence of triangle meshes, ACM Transactions on Graphics, 27:3(2008), pp. 1-11.

[44] Computational conformal geometry library 1.1, http://www.cs.sunysb.edu/ ${ }^{\sim}$ manifold/CCGL1.1/.

[45] W. Zeng, M. Jin, F. Luo, And X. Gu, Computing canonical homotopy class representative using hyperbolic structure, in: IEEE International Conference on Shape Modeling and Applications (SMI'09), 2009.

[46] W. P. Thurston, Geometry and Topology of Three-Manifolds. Princeton lecture notes, 1976.

[47] F. Luo, X. Gu, ANd J. DAI, Variational Principles for Discrete Surfaces, ser. Advanced Lectures in Mathematics. High Education Press and International Press, 2007.

[48] W. Zeng, F. LuO, S.-T. YAU, And X. Gu, Surface quasi-conformal mapping by solving beltrami equations, in: IMA: 13th International Conference on The Mathematics of Surfaces, 2009.

[49] W. Zeng, L. M. Lui, X. Gu, and S.-T. YAU, Shape analysis by conformal modules, in: Methods and Applications of Analysis, 2009.

[50] M. Jin, W. Zeng, N. Ding, AND X. Gu, Computing fenchel-nielsen coordinates in teichmuller shape space, in: IEEE International Conference on Shape Modeling and Applications (SMI'09), 2009. 
